\title{
ANALYTIC CRACK SOLUTIONS FOR TUT FIELDS AROUND HYDRAULIC FRACTURES
}

\section{N. R. Warpinski}

Sandia National Laboratories, Albuquerque,.NM

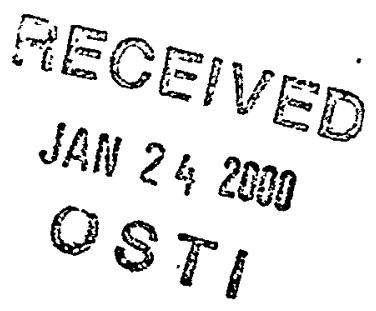

\begin{abstract}
The recent development of downhole tiltmeter arrays for monitoring hydraulic fractures has provided new information on fracture growth and geometry. These downhole arrays offer the significant advantages of being close to the fracture (large signal) and being unaffected by the free surface. As with surface tiltmeter data, analysis of these measurements requires the inversion of a crack or dislocation model. To supplement the dislocation models of Davis [1983], Okada [1992] and others, this work has extended several elastic crack solutions to provide tilt calculations. The solutions include constant-pressure $2 \mathrm{D}$, penny-shaped, and 3Delliptic cracks and a 2D-variable-pressure crack. Equations are developed for an arbitrary inclined fracture in an infinite elastic space. Effects of fracture height, fracture length, fracture dip, fracture azimuth, fracture width and monitoring distance on the tilt distribution are given, as well as comparisons with the dislocation model. The results show that the tilt measurements are very sensitive to the fracture dimensions, but also that it is difficult to separate the competing effects of the various parameters.
\end{abstract}




\section{DISCLAIMER}

This report was prepared as an account of work sponsored by an agency of the United States Government. Neither the United States Government nor any agency thereof, nor any of their employees, make any warranty, express or implied, or assumes any legal liability or responsibility for the accuracy, completeness, or usefuiness of any information, apparatus, product, or process disclosed, or represents that its use would not infringe privately owned rights. Reference herein to any specific commercial product, process, or service by trade name, trademark, manufacturer, or otherwise does not necessarily constitute or imply its endorsement, recommendation, or favoring by the United States Government or any agency thereof. The views and opinions of authors expressed herein do not necessarily state or reflect those of the United States Government or any agency thereof. 


\section{DISCLAIMER}

\section{Portions of this document may be illegible in electronic image products. Images are produced from the best available original document.}




\section{Introduction}

Surface tiltmeter arrays have been used extensively for monitoring the azimuth and dip of hydraulic fractures and the overall inflation of magmatic channels around volcanoes. Recently, however, testing at the Gas Research Institute /Department Of Energy (GRI/DOE) Multi-Site facility near Rifle Colorado has shown that downhole tiltmeters could provide useful information about other fracture parameters [Warpinski, 1994 \& 1996, Peterson et al., 1996, Branagan et al., 1996, Warpinski et al., 1997a \& 1997b] and such" monitoring is now available as a service [Wright, 1998 ; Wright et al., 1998]. In both cases - surface and downhole - it is necessary to invert the measured tilt data to extract meaningful information about the fracture. The inversion process requires a model of the tilt field induced by a pressurized fracture.

Models for evaluating surface tilts have generally been obtained from either elastic crack solutions [e.g., Sun, 1969; Pollard and Holzhausen, 1979] or dislocation solutions [e.g., Davis, 1983; Okada, 1992]. The Davis [1983] formulation is particularly attractive for surface tilts because of its simplicity, but the Okada [1992] results are more complete and provide both surface and internal deformations and tilts. Both of these models apply a rectangular dislocation of uniform width to produce the deformation associated with a dilated fracture. Davis [1983] notes that the rectangular dislocation is an approximation of a true tensile fracture, but the deformation far from the fracture should be accurate. The deformation close to the fracture, however, is likely to be considerably in error. Furthermore, there is no direct tie to the internal pressure within the hydraulic fracture, which is usually the only parameter measured.

The purpose of this study was to develop exact analytical solutions of the tilt fields around pressurized cracks in elastic bodies that could be used as an alternate to the dislocation solution. These new results can be used to assess the applicability of the dislocation model as a function of 
distance from the fracture and assess the coupling of the pressure to the dislocation inversion. No new crack models have been formulated, but rather classical solutions to constant-pressure 2D, penny-shaped, and 3D elliptical cracks and a non-constant-pressure 2D crack have been reworked to obtain tilt equations in addition to the stress and displacement equations.

These results also provide new tools that can be used to evaluate the sensitivity of the tilt measurements to the various parameters and the ability of an inversion procedure to adequately distinguish between competing parameters. Any of these models can be used directly in an inversion algorithm, although the 3D elliptic crack is the most versatile and would probably be most useful for practical applications.

\section{Models}

Solutions for four crack models are applied to the problem of the downhole tilt field generated by the presence of a pressurized crack. These models include a plane 2D crack, an axisymmetric radial crack (penny-shaped), and a 3D flat elliptical crack for the constant-pressure case and a 2D plane crack for a non-constant-pressure solution. In all these cases it will be assumed that the rock mass is homogeneous, isotropic and elastic.

\subsection{2-D Model}

The simplest analytic solution for the tilt field around a fracture is that of an infinitely long 2-D crack, a solution that was developed by Westergaard [1939], Sneddon and Elliot [1946], and Sneddon [1946]. The geometry for this crack is shown in Figure 1, which follows directly from Sneddon and Elliot [1946] and Sneddon's [1946] nomenclature and orientation. The geometry is rotated from Sneddon's [1946] so that the crack height is $2 c$, and all locations are measured by 
the three distance parameters, $r, r_{1}$, and $r_{2}$, and the three angles $\theta, \theta_{1}$, and $\theta_{2}$. The crack is uniformly pressurized at a level $\mathrm{P}$ in a material having a Young's modulus, E, and Poisson's ratio, $v$. Note that Sneddon's [1946] original coordinates having the $\mathrm{x}$ direction aligned with the crack height and the y direction normal to the crack is somewhat contrary to the normal geometry of a vertical slice of a $2 \mathrm{D}$ vertical fracture. However, those coordinates are kept here to facilitate references to the original work.

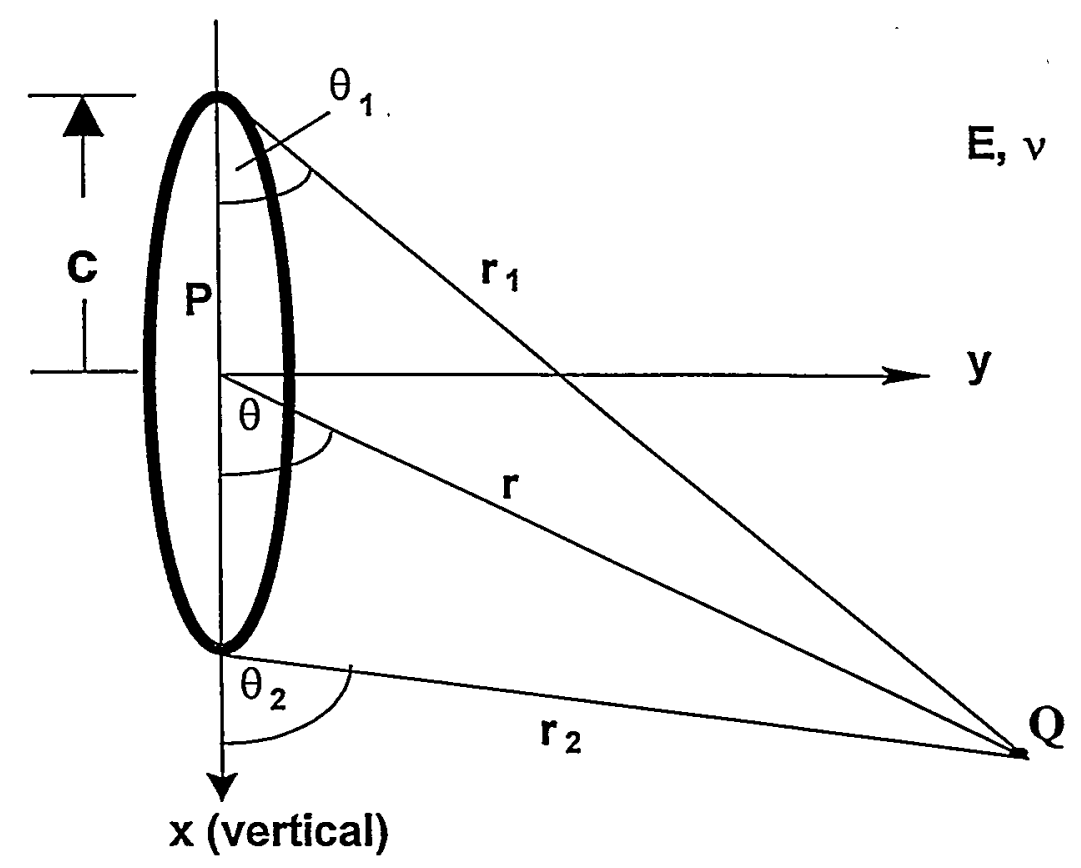

Figure 1. Geometry of a 2-D crack

The basic solution is due to Westergaard [1939] in terms of a stress function, Z, given as

$$
Z=P\left[\frac{z}{\sqrt{z^{2}-c^{2}}}-1\right]
$$

where $z$ is the complex variable, $z=x+i y$. Sneddon [1946] gives equations for the stress field around the crack, but only the displacements $\left(u_{x}, u_{y}\right)$ are of interest here and these are given by 


$$
\frac{2 E}{(1+v)} u_{x}=(1-2 v) \operatorname{Re}[\bar{Z}]-y \operatorname{Im}[z]
$$

and

$$
\frac{2 E}{(1+v)} u_{y}=2(1-v) \operatorname{Im}[\bar{Z}]-y \operatorname{Re}[Z]
$$

where the overbar represents the integral of the stress function $Z$ with respect to $z$. The tilt vector can be separated into two components, one orthogonal to the face of the fracture and one parallel to the face. Upon differentiation, the tilt field orthogonal to the fracture can be deduced as

$$
\frac{\partial u_{y}}{\partial x}=\frac{(1+v)}{E}\left\{2(1-v) \operatorname{Im}[Z]-y \operatorname{Re}\left[Z^{\prime}\right]\right\}
$$

while other derivatives that will be useful for inclined fractures are given by

$$
\begin{gathered}
\frac{\partial u_{x}}{\partial x}=\frac{(1+v)}{E}\left\{(1-2 v) \operatorname{Re}[Z]-y \operatorname{Im}\left[Z^{\prime}\right]\right\}, \\
\frac{\partial u_{x}}{\partial y}=\frac{(1+v)}{E}\left\{-2(1-v) \operatorname{Im}[Z]-y \operatorname{Re}\left[Z^{\prime}\right]\right\},
\end{gathered}
$$

and

$$
\frac{\partial u_{y}}{\partial y}=\frac{(1+v)}{E}\left\{(1-2 v) \operatorname{Re}[Z]+y \operatorname{Im}\left[Z^{\prime}\right]\right\}
$$

When $Z$ is replaced, the derivatives can be written as

$$
\begin{gathered}
\frac{\partial u_{y}}{\partial x}=\frac{2(1+v) P}{E}\left\{(1-v) \frac{r}{r_{1} r_{2}} \sin \left(\theta-\frac{1}{2} \theta_{1}-\frac{1}{2} \theta_{2}\right)+\frac{r \sin \theta}{2 c}\left[\frac{c^{2}}{r_{1} r_{2}}\right]^{\frac{3}{2}} \cos \left(\frac{3}{2} \theta_{1}+\frac{3}{2} \theta_{2}\right)\right\}, \\
\frac{\partial u_{x}}{\partial x}=\frac{(1+v) P}{E}\left\{(1-2 v)\left[\frac{r}{r_{1} r_{2}} \cos \left(\theta-\frac{1}{2} \theta_{1}-\frac{1}{2} \theta_{2}\right)-1\right]-\frac{r \sin \theta}{c}\left[\frac{c^{2}}{r_{1} r_{2}}\right]^{\frac{3}{2}} \sin \left(\frac{3}{2} \theta_{1}+\frac{3}{2} \theta_{2}\right)\right\},
\end{gathered}
$$




$$
\frac{\partial u_{x}}{\partial y}=\frac{2(1+v) P}{E}\left\{-(1-v) \frac{r}{r_{1} r_{2}} \sin \left(\theta-\frac{1}{2} \theta_{1}-\frac{1}{2} \theta_{2}\right)+\frac{r \sin \theta}{2 c}\left[\frac{c^{2}}{r_{1} r_{2}}\right]^{\frac{3}{2}} \cos \left(\frac{3}{2} \theta_{1}+\frac{3}{2} \theta_{2}\right)\right\}
$$

and

$$
\frac{\partial u_{y}}{\partial y}=\frac{(1+v) P}{E}\left\{(1-2 v)\left[\frac{r}{r_{1} r_{2}} \cos \left(\theta-\frac{1}{2} \theta_{1}-\frac{1}{2} \theta_{2}\right)-1\right]+\frac{r \sin \theta}{c}\left[\frac{c^{2}}{r_{1} r_{2}}\right]^{\frac{3}{2}} \sin \left(\frac{3}{2} \theta_{1}+\frac{3}{2} \theta_{2}\right)\right\}
$$

The first of these four equations can be used directly to give the tilt component orthogonal to a vertical fracture that is monitored in an offset well using a vertical tilt array. The tilt component parallel to the fracture (out of the plane) is zero since the crack is infinitely long.

For fractures with dip, it is necessary to rotate the displacement gradients into the correct orientation and it is necessary to find the correct spatial parameters. Considering the transformation first, Figure 2 shows the fracture with dip and the observation well and then a separate rotated view of this system. It is observed that the displacement of interest, the one normal to the borehole $\left(u_{n}\right)$, is given by

$$
u_{n}=u_{y} \cos \gamma+u_{x} \sin \gamma,
$$

where $\gamma$ is the angle of the fracture plane referenced to the vertical (e.g., zero is a vertical fracture). The tiltmeter array measures the variation of the derivatives of this displacement along the s direction,

$$
\frac{\partial u_{n}}{\partial s}=\frac{\partial u_{n}}{\partial y} \frac{d y}{d s}+\frac{\partial u_{n}}{\partial x} \frac{d x}{d s}
$$



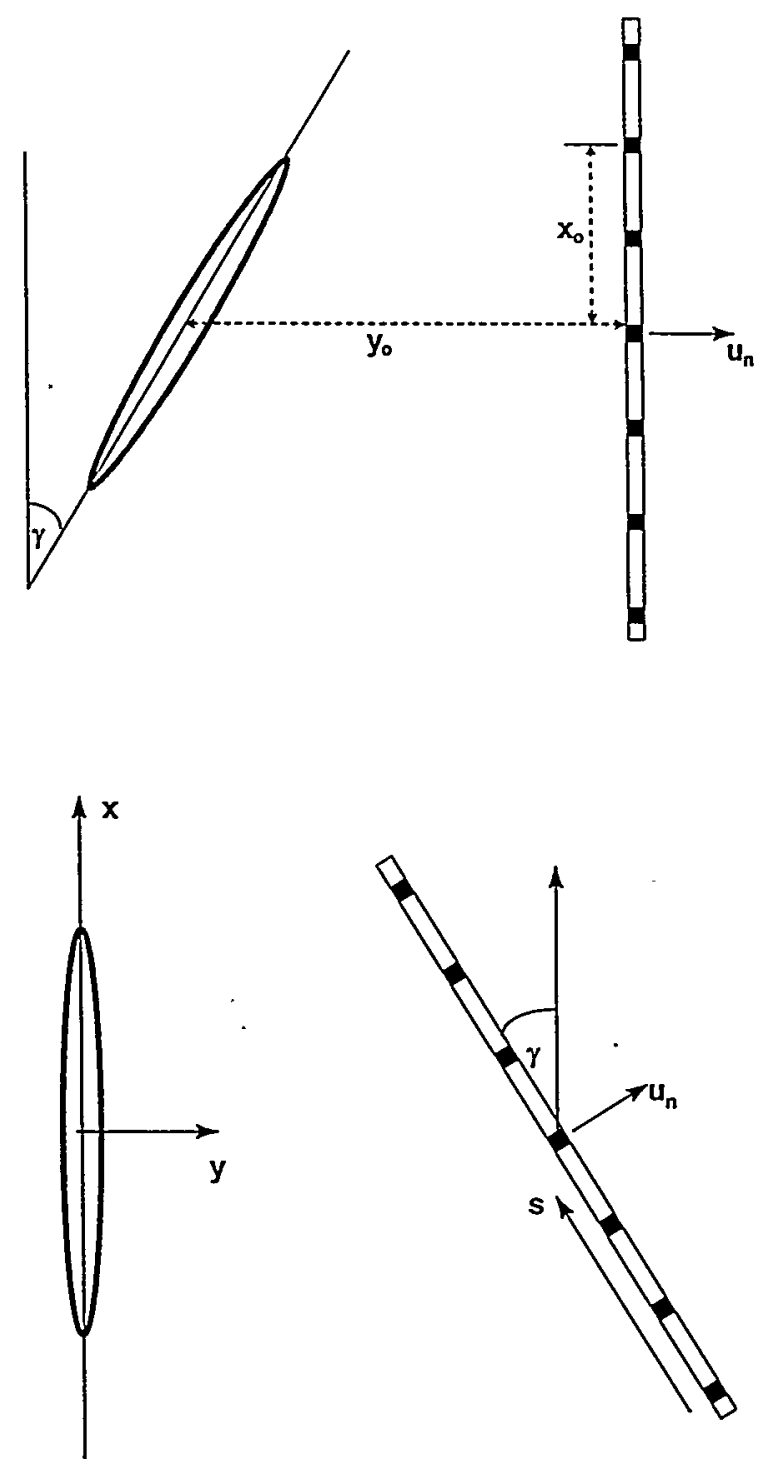

Figure 2. Geometry for 2D fracture with dip.

Since the two spatial derivatives are given by

$$
\frac{d y}{d s}=-\sin \gamma \text { and } \frac{d x}{d s}=\cos \gamma,
$$

the tilt can be reduced to

$$
\frac{\partial u_{n}}{\partial s}=\sin \gamma \cos \gamma\left[-\frac{\partial u_{y}}{\partial y}+\frac{\partial u_{x}}{\partial x}\right]-\sin ^{2} \gamma \frac{\partial u_{x}}{\partial y}+\cos ^{2} \gamma \frac{\partial u_{y}}{\partial x}
$$


The final step is to determine the correct geometric parameters to characterize the distance from the fracture to the tiltmeters. Referring to the unrotated coordinate system in Figure 2, it can be found from geometry considerations that

$$
\hat{x}=y_{o} \sin \gamma+x_{o} \cos \gamma
$$

and

$$
\hat{y}=y_{0} \cos \gamma-x_{0} \sin \gamma
$$

where the variables with hats are the distances to use in the analysis. These equations complete the analysis of downhole tilt data for any $2 \mathrm{D}$ fracture, as long as it is far from the free surface.

\subsection{Penny-Shaped Model}

For a penny-shaped crack, as shown in Figure 3, the problem is tractable because of the axisymmetry, but is considerably more complicated than the 2-D crack. Sneddon [1946] found solutions for stresses and displacements in terms of Bessel-function arguments, although only displacements are needed here.

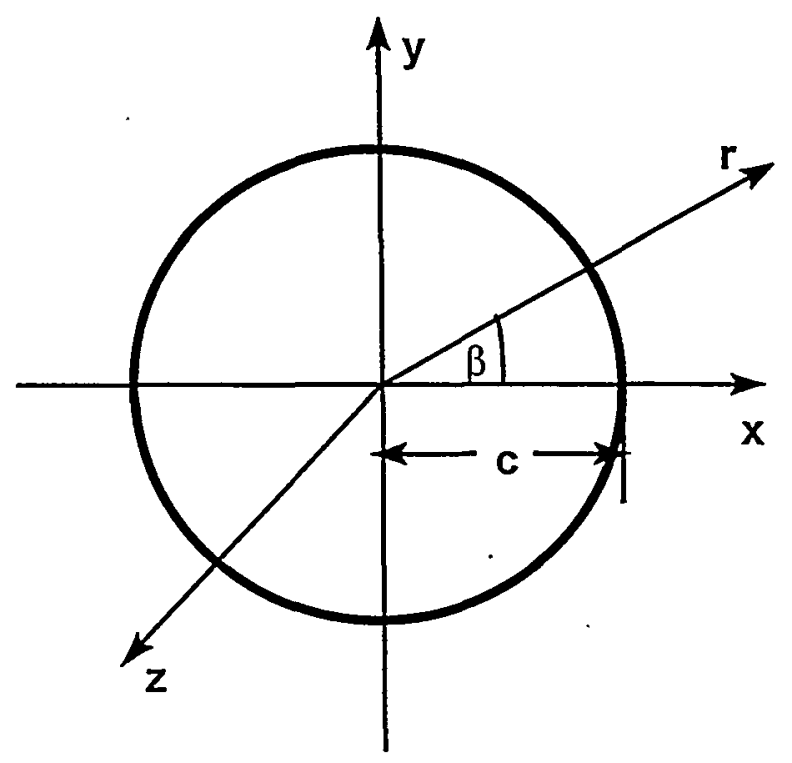

Figure 3. Geometry of a penny-shaped crack 
By adopting the following notation,

$$
\begin{aligned}
& \zeta=z / c \\
& \rho=r / c \\
& r^{2}=1+\zeta^{2} \\
& R^{2}=\left(\rho^{2}+\zeta^{2}-1\right)^{2}+4 \zeta^{2} \\
& \zeta \tan \theta=1 \\
& 2 \zeta \cot \varphi=\rho^{2}+\zeta^{2}-1
\end{aligned}
$$

Sneddon [1946] showed that the displacements are given as the following integrals,

$$
u_{r}=\frac{2 P c(1-v)}{\pi E} \int_{b}^{\infty}(1-2 v-\zeta \eta) \frac{d}{d \eta}\left(\frac{\sin \eta}{\eta}\right) e^{-\zeta \eta} J_{1}(\rho \eta) d \eta
$$

and

$$
u_{z}=-\frac{4 P c\left(1-v^{2}\right)}{\pi E} \int_{b}^{\infty}\left(1+\frac{\zeta \eta}{2(1-v)}\right) \frac{d}{d \eta}\left(\frac{\sin \eta}{\eta}\right) e^{-\zeta \eta} J_{0}(\rho \eta) d \eta
$$

By rearranging and defining the functions $C_{n}^{m}$ and $S_{n}^{m}$ as

$$
C_{n}^{m}(\rho, \zeta)=\int_{0}^{\infty} \eta^{n-1} e^{-\zeta \eta} J_{m}(\rho \eta) \cos \eta d \eta
$$

and

$$
S_{n}^{m}(\rho, \zeta)=\int_{0}^{\infty} \eta^{n-1} e^{-\zeta \eta} J_{m}(\rho \eta) \sin \eta d \eta,
$$

the integrations can be performed and the displacements can be written in terms of elementary functions given by 


$$
\begin{array}{ll}
C_{1}^{0}=\frac{1}{\sqrt{R}} \cos \left(\frac{\varphi}{2}\right) & S_{1}^{0}=\frac{1}{\sqrt{R}} \sin \left(\frac{\varphi}{2}\right) \\
C_{2}^{0}=\frac{r}{R \sqrt{R}} \cos \left(\frac{3 \varphi}{2}-\theta\right) & S_{2}^{0}=\frac{r}{R \sqrt{R}} \sin \left(\frac{3 \varphi}{2}-\theta\right) \\
C_{0}^{1}=\frac{1}{\rho}\left[\sqrt{R} \cos \left(\frac{\varphi}{2}\right)-\zeta\right] & S_{0}^{1}=\frac{1}{\rho}\left[1-\sqrt{R} \sin \left(\frac{\varphi}{2}\right)\right] \\
C_{1}^{1}=\frac{1}{\rho}-\frac{r}{\rho \sqrt{R}} \cos \left(\theta-\frac{\varphi}{2}\right) & S_{1}^{1}=\frac{r}{\rho \sqrt{R}} \sin \left(\theta-\frac{\varphi}{2}\right) \\
C_{2}^{1}=\frac{\rho R}{\sqrt{R}} \cos \left(\frac{3 \varphi}{2}\right) & S_{2}^{1}=\frac{\rho R}{\sqrt{R}} \sin \left(\frac{3 \varphi}{2}\right) \\
C_{1}^{2}=\frac{2}{\rho} C_{0}^{1}-C_{1}^{0} & S_{1}^{2}=\frac{2}{\rho} S_{0}^{1}-S_{1}^{0} \\
C_{2}^{2}=\frac{2}{\rho} C_{1}^{1}-C_{2}^{0} &
\end{array}
$$

and

$$
S_{0}^{0}=\tan ^{-1}\left[\frac{\sqrt{R} \sin \left(\frac{\bar{\theta}}{2}\right)+r \sin \theta}{\sqrt{R} \cos \left(\frac{\theta}{2}\right)+r \cos \theta}\right], \rho \neq 0 \quad S_{0}^{2}=\frac{1}{\rho}\left(C_{0}^{1}-\zeta S_{0}^{1}\right)
$$

To find the tilts around a vertical fracture, the displacement and the gradients of the displacements are needed and these are given by

$$
\begin{aligned}
& u_{r}=\frac{2 P c(1+v)}{\pi E}\left\{(1-2 v)\left[C_{0}^{1}-\frac{\rho}{2}\left(S_{0}^{0}+S_{0}^{2}\right)\right]-\zeta\left[C_{1}^{1}-S_{0}^{1}\right]\right\} \\
& \frac{\partial u_{r}}{\partial r}=\frac{P(1+v)}{\pi E}\left\{(1-2 v)\left[C_{1}^{0}-C_{1}^{2}-S_{0}^{0}+S_{0}^{2}\right]-\zeta\left[C_{2}^{0}-C_{2}^{2}-S_{1}^{0}+S_{1}^{2}\right]\right\} \\
& \frac{\partial u_{z}}{\partial r}=\frac{4 P\left(1-v^{2}\right)}{\pi E}\left\{C_{1}^{1}-S_{0}^{1}+\frac{\zeta}{2(1-v)}\left[C_{2}^{1}-S_{1}^{1}\right]\right\}
\end{aligned}
$$

From these terms, the components of tilt normal and parallel to the fracture face can be found as 


$$
\begin{aligned}
& \frac{\partial u_{z}}{\partial y}=\sin \beta \frac{\partial u_{z}}{\partial r} \\
& \frac{\partial u_{h}}{\partial y}=\cos \beta \sin \beta\left\{\frac{\partial u_{r}}{\partial r}-\frac{u_{r}}{r}\right\}
\end{aligned}
$$

where $\beta$ is the angle from the center of the crack to the $x, y$ position of the measurement station and $\mathrm{r}$ is the distance from the center of the crack to the measurement station.

For a fracture with dip, additional derivatives are needed and are found as

$$
\frac{\partial u_{r}}{\partial z}=\frac{-2 P(1+v)}{\pi E}\left\{C_{1}^{1}-S_{0}^{1}-\zeta\left[C_{2}^{1}-S_{1}^{1}\right]-(1-2 v)\left[C_{1}^{1}-S_{0}^{1}\right]\right\}
$$

and

$$
\frac{\partial u_{z}}{\partial z}=\frac{4 P\left(1-v^{2}\right)}{\pi E}\left\{C_{1}^{0}-S_{0}^{0}+\frac{1}{2(1-v)}\left[-C_{1}^{0}-S_{0}^{0}+\zeta\left(C_{2}^{0}-S_{1}^{0}\right)\right]\right\}
$$

For fractures with dip, it is necessary to rotate the displacement gradients into the correct orientation and it is necessary to find the correct spatial parameters. Figure 4 shows the fracture with dip and the observation well and then the rotated view of this geometry. It is observed that the two displacements of interest, the one normal to the fracture $\left(u_{n}\right)$ and the one parallel to the fracture $\left(u_{p}\right)$ are given by

$$
\begin{gathered}
u_{n}=u_{z} \cos \gamma+\left(u_{r} \sin \beta\right) \sin \gamma \\
u_{p}=u_{r} \cos \beta,
\end{gathered}
$$

where $\gamma$ is the angle of the fracture plane referenced to the vertical (e.g., zero is a vertical fracture) and $\beta$ is the reference angle to the measurement station (e.g., Figure 3). The tiltmeter array measures the variation of the derivatives of these displacements along the s direction,

$$
\frac{\partial u_{n}}{\partial s}=\frac{\partial u_{n}}{\partial z} \frac{d z}{d s}+\frac{\partial u_{n}}{\partial y} \frac{d y}{d s}
$$


and

$$
\frac{\partial u_{p}}{\partial s}=\frac{\partial u_{p}}{\partial z} \frac{d z}{d s}+\frac{\partial u_{p}}{\partial y} \frac{d y}{d s} .
$$
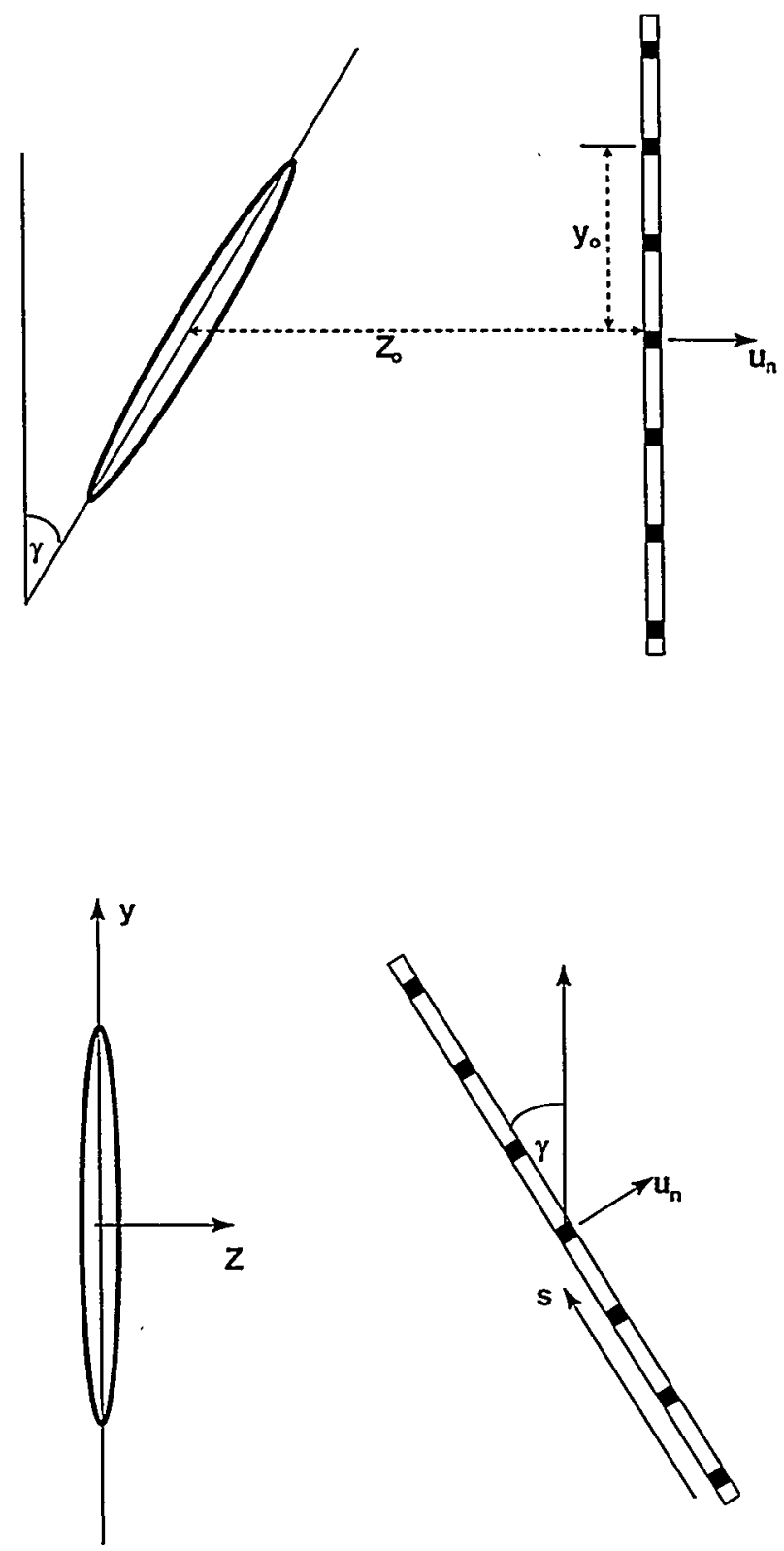

Figure 4. Geometry for penny-shaped fracture with dip. 
Since the two spatial derivatives are given by

$$
\frac{d z}{d s}=-\sin \gamma \text { and } \frac{d y}{d s}=\cos \gamma
$$

the tilt derivatives can be reduced to

$$
\frac{\partial u_{n}}{\partial s}=\sin \gamma \cos \gamma\left[-\frac{\partial u_{z}}{\partial z}+\sin ^{2} \beta \frac{\partial u_{r}}{\partial r}-\cos ^{2} \beta \frac{u_{r}}{r}\right]-\sin ^{2} \gamma \sin \beta \frac{\partial u_{r}}{\partial z}+\cos ^{2} \gamma \sin \beta \frac{\partial u_{z}}{\partial r}
$$

and

$$
\frac{\partial u_{p}}{\partial s}=-\sin \gamma \cos \beta \frac{\partial u_{r}}{\partial z}+\cos \gamma \cos \beta \sin \beta\left[\frac{\partial u_{r}}{\partial r}-\frac{u_{r}}{r}\right]
$$

The final task is to determine the correct geometric parameters to characterize the distance from the fracture to the tiltmeters. Referring to the unrotated schematic in Figure 4, it can be found from geometry considerations that

$$
\begin{gathered}
\hat{x}=x \\
\hat{y}=Z_{o} \sin \gamma+y_{o} \cos \gamma=Z_{o} \sin \gamma+r_{o} \cos \gamma \sin \beta
\end{gathered}
$$

and

$$
\hat{z}=z_{o} \cos \gamma-y_{o} \sin \gamma=z_{o} \cos \gamma-r_{o} \sin \gamma \sin \beta,
$$

so that

$$
\hat{r}=\sqrt{\hat{x}^{2}+\hat{y}^{2}} \text { and } \hat{\beta}=\tan ^{-1}\left(\frac{\hat{y}}{\hat{x}}\right)
$$

where the variables with hats are the correct distance and angle to use in the analysis. These equations complete the analysis of downhole tilt data for any penny-shaped fracture, as long as it is far from the free surface. 


\subsection{Flat Elliptic Crack Model}

The case of a 3D flat elliptic crack will be developed in more detail here because it is the most general model, it is considerably more complex, and it was not as well developed in the original papers. Considering a 3-dimensional flat elliptic crack opened by internal pressure and having the geometry shown in Figure 5, Green and Sneddon [1950] found an analytical solution for the following assumptions:

- infinite medium

- homogeneous isotropic material

- linear elastic behavior

- uniform pressure

- length $>$ height $(a>b)$.

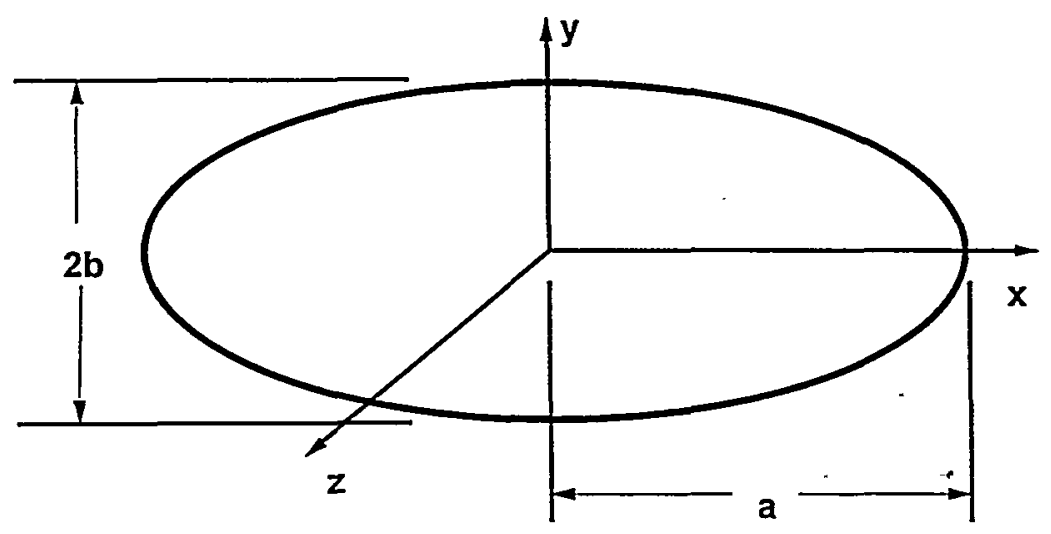

Figure 5. Geometry of fracture for tilt and stress calculations

Given these restrictions, the displacements and stresses can be given by

$$
D=8 \frac{\partial}{\partial \bar{z}}\left\{(1+2 \eta) \phi+Z \frac{\partial \phi}{\partial Z}\right\}
$$




$$
\begin{gathered}
w=-8(1-\eta) \frac{\partial \phi}{\partial Z}+4 Z \frac{\partial^{2} \phi}{\partial Z^{2}}, \\
\Theta=-8 G\left\{(1-2 \eta) \frac{\partial^{2} \phi}{\partial Z^{2}}+Z \frac{\partial^{3} \phi}{\partial Z^{3}}\right\}, \\
\Phi=32 G \frac{\partial^{2}}{\partial^{-2}}\left\{(1-2 \eta) \phi+Z \frac{\partial \phi}{\partial Z}\right\}, \\
\sigma_{Z}=-8 G \frac{\partial^{2} \phi}{\partial Z^{2}}+8 G Z \frac{\partial^{3} \phi}{\partial Z^{3}}, \text { and } \\
\Psi=16 G Z \frac{\partial^{3} \phi}{\partial z \partial Z^{2}},
\end{gathered}
$$

with

$$
\begin{gathered}
D=\tilde{u}+i v, \\
\Theta=\sigma_{x}+\sigma_{y}, \\
\Phi=\sigma_{x}-\sigma_{y}+2 i \tau_{x y}, \text { and } \\
\Psi=\tau_{x z}+i \tau_{y z} .
\end{gathered}
$$

In these equations, $\phi$ is the solution stress function, $G$ is the shear modulus of the material and $\eta$ is Poisson's ratio. Additionally, $Z$ is the third coordinate while $z$ is the complex variable given by $z=x+i y$ and $\bar{z}$ is its complex conjugate. Green and Sneddon [1950] found a solution of the problem by converting to an ellipsoidal coordinate system, $\lambda, \mu, \nu$, given by

$$
\begin{aligned}
& a^{2}\left(a^{2}-b^{2}\right) x^{2}=\left(a^{2}+\lambda\right)\left(a^{2}+\mu\right)\left(a^{2}+v\right) \\
& b^{2}\left(b^{2}-a^{2}\right) y^{2}=\left(b^{2}+\lambda\right)\left(b^{2}+\mu\right)\left(b^{2}+v\right) \\
& a^{2} b^{2} Z^{2}=\lambda \mu \nu
\end{aligned}
$$

where 


$$
\infty>\lambda \geq 0 \geq \mu \geq-b^{2} \geq v \geq-a^{2} .
$$

In this coordinate system, the solution can be found as an integration of combined coordinates

as

$$
\phi=\frac{a b^{2} p}{32 G E(k)} \int_{\lambda}^{\infty}\left\{\frac{x^{2}}{a^{2}+s}+\frac{y^{2}}{b^{2}+s}+\frac{Z^{2}}{s}-1\right\} \frac{d s}{\sqrt{s\left(a^{2}+s\right)\left(b^{2}+s\right)}},
$$

where $E(k)$ is a complete elliptic integral of the second kind of modulus $k$, with

$$
k=\frac{\sqrt{a^{2}-b^{2}}}{a} .
$$

\subsection{Application to a Vertical Fracture (Length $>$ Height)}

The tilts normal to the face of the fracture can be found as

$$
\begin{aligned}
\frac{\partial w}{\partial y} & =\frac{-8 A Z}{a b^{2}}\left[-(1-2 v)\left\{k^{\prime 2} \frac{\operatorname{sn}^{2} u}{\mathrm{cn}^{2} u}\right\} \frac{d u}{d \lambda} \frac{\partial \lambda}{\partial y}\right] \\
& +Z\left\{2 k^{\prime 2} \frac{\operatorname{sn} u \operatorname{dn} u}{\mathrm{cn}^{3} u}\right\}\left(\frac{d u}{d \lambda}\right)^{2} \frac{\partial \lambda}{\partial y} \frac{\partial \lambda}{\partial Z} \\
& \left.+Z\left\{k^{\prime 2} \frac{\operatorname{sn}^{2} u}{\operatorname{cn}^{2} u}\right\}\left(\frac{d^{2} u}{d \lambda^{2}} \frac{\partial \lambda}{\partial y} \frac{\partial \lambda}{\partial Z}+\frac{d u}{d \lambda} \frac{\partial^{2} \lambda}{\partial y \partial Z}\right)\right],
\end{aligned}
$$

where sn, dn and cn are Jacobian elliptic functions, $A$ is given by

$$
A=-\frac{a b^{2} p}{16 G E(k)},
$$

and $u$ is defined as

$$
\lambda=a^{2} \frac{\operatorname{cn} u}{\sin u}
$$

The derivatives in the tilt equation are found from 


$$
\begin{aligned}
& \frac{d u}{d \lambda}=\frac{-\operatorname{sn}^{3} u}{2 a^{2} \operatorname{cn} u \operatorname{dn} u} \\
& \frac{d^{2} u}{d \lambda^{2}}=\frac{-\operatorname{sn}^{2} u}{2 a^{2}}\left[3+\frac{\operatorname{sn}^{2} u}{\operatorname{cn}^{2} u}+k^{2} \frac{\operatorname{sn}^{2} u}{\operatorname{dn}^{2} u}\right] \frac{d u}{d \lambda}, \\
& \frac{\partial \lambda}{\partial y}=\frac{2 y \lambda\left(a^{2}+\lambda\right)}{(\lambda-\mu)(\lambda-v)}, \\
& \frac{\partial \lambda}{\partial Z}=\frac{2 Z\left(a^{2}+\lambda\right)\left(b^{2}+\lambda\right)}{(\lambda-\mu)(\lambda-\nu)} \\
& \frac{\partial^{2} \lambda}{\partial y \partial Z}=\frac{y Z}{4\left(h_{1}^{2}\right)^{2} \lambda\left(b^{2}+\lambda\right)}\left[-\frac{1}{\lambda}-\frac{1}{b^{2}+\lambda}+\frac{1}{2 h_{1}^{2}}\left\{\frac{x^{2}}{\left(a^{2}+\lambda\right)^{3}}+\frac{y^{2}}{\left(b^{2}+\lambda\right)^{3}}+\frac{Z^{2}}{\lambda^{3}}\right\}\right],
\end{aligned}
$$

and

$$
h_{1}^{2}=\frac{(\lambda-\mu)(\lambda-v)}{4 \lambda\left(a^{2}+\lambda\right)\left(b^{2}+\lambda\right)} .
$$

More information about these derivatives and other characteristics of the ellipsoidal confocal coordinate system can be found in Whittaker and Watson [1927]. This same reference has extensive information about the Jacobian elliptic functions, as does Abramowitz and Stegun [1970]. In addition, Sih and Liebowitz [1968] provide some discussion on the 3D-elliptic-crack solution that is useful.

The tilts parallel to the fracture face are found from

$$
\begin{aligned}
\frac{\partial \tilde{u}}{\partial y} & =\frac{8(1-2 \eta) A x}{a^{3}}\left\{\operatorname{sn}^{2} u\right\} \frac{d u}{d \lambda} \frac{\partial \lambda}{\partial y} \\
& +\frac{8 A Z^{2}}{a b^{2}}\left[\left\{2 k^{\prime 2} \frac{\operatorname{sn} u \operatorname{dn} u}{\mathrm{cn}^{3} u}\right\}\left(\frac{d u}{d \lambda}\right)^{2} \frac{\partial \lambda}{\partial x} \frac{\partial \lambda}{\partial y}\right. \\
& \left.+\left\{k^{\prime 2} \frac{\mathrm{sn}^{2} u}{\mathrm{cn}^{2} u}\right\}\left\{\frac{d^{2} u}{d \lambda^{2}} \frac{\partial \lambda}{\partial x} \frac{\partial \lambda}{\partial y}+\frac{d u}{d \lambda} \frac{\partial^{2} \lambda}{\partial x \partial y}\right\}\right]
\end{aligned}
$$


where the additional derivatives are given by

$$
\frac{\partial \lambda}{\partial x}=\frac{2 x \lambda\left(b^{2}+\lambda\right)}{(\lambda-\mu)(\lambda-v)}
$$

and

$$
\frac{\partial^{2} \lambda}{\partial x \partial y}=\frac{x y}{4\left(h_{1}^{2}\right)^{2}\left(a^{2}+\lambda\right)\left(b^{2}+\lambda\right)}\left[-\frac{a^{2}+b^{2}+2 \lambda}{\left(a^{2}+\lambda\right)\left(b^{2}+\lambda\right)}+\frac{1}{2 h_{1}^{2}}\left\{\frac{x^{2}}{\left(a^{2}+\lambda\right)^{3}}+\frac{y^{2}}{\left(b^{2}+\lambda\right)^{3}}+\frac{Z^{2}}{\lambda^{3}}\right\}\right] .
$$

The calculation of the $\lambda, \mu, \nu$ coordinates requires the solution of the cubic equation

$$
\lambda^{3}+\lambda^{2}\left(a^{2}+b^{2}-x^{2}-y^{2}-Z^{2}\right)+\lambda\left(a^{2} b^{2}-b^{2} x^{2}-a^{2} y^{2}-a^{2} Z^{2}-b^{2} Z^{2}\right)-a^{2} b^{2} Z^{2}=0
$$

for $\lambda$, followed by solution of the quadratic equation

$$
\mu^{2}\left(a^{2} \lambda+\lambda\right)+\mu\left(a^{2} b^{2} Z^{2}+b^{2} \lambda Z^{2}+a^{4} \lambda+a^{2} \lambda^{2}-a^{2} \lambda x^{2}+b^{2} \lambda x^{2}\right)+a^{4} b^{2} Z^{2}+a^{2} b^{2} \lambda Z^{2}=0
$$

for $\mu$, and then

$$
\nu=\frac{a^{2} b^{2} Z^{2}}{\lambda \mu}
$$

The procedure for using these equations is as follows:

1. Select point $x, y, Z$ for which the calculation is to be made

2. Determine the appropriate $\lambda, \mu, v$ for this point

3. Determine the value of $u$

4. Obtain tilts.

One confusing point is that Green and Sneddon [1950] used $u$ for both the displacement in the $\mathrm{x}$ direction as well as for a transformed function. To help eliminate confusion in this paper, $\widetilde{u}$ has been used to denote the displacement. The only place the displacement enters is through the original complex equations and in the definition of the tilt. 


\subsubsection{Non-Vertical Fracture or Height Greater than Length}

The previous solution is for a vertical fracture whose height is greater than its length, which is a quite limiting constraint. However, this model can be used to also extract the tilts for a fracture with dip and for one whose height is greater than its length. To obtain the tilts for these cases, it is necessary to obtain the displacement derivatives for the seven other components. These are given as:

$$
\begin{aligned}
& \frac{\partial \tilde{u}}{\partial x}=\frac{8(1-2 \eta) A}{a^{3} k^{2}}\{u-E(u)\} \\
& +\frac{8(1-2 \eta) A x}{a^{3}}\left\{\operatorname{sn}^{2} u\right\} \frac{d u}{d \lambda} \frac{\partial \lambda}{\partial x} \\
& +\frac{8 A Z^{2}}{a b^{2}}\left[\left\{2 k^{\prime 2} \frac{\sin u \operatorname{dn} u}{\mathrm{cn}^{3} u}\right\}\left(\frac{d u}{d \lambda}\right)^{2}\left(\frac{\partial \lambda}{\partial x}\right)^{2}\right. \\
& \left.+\left\{k^{\prime 2} \cdot \frac{\operatorname{sn}^{2} u}{\operatorname{cn}^{2} u}\right\}\left\{\frac{d^{2} u}{d \lambda^{2}}\left(\frac{\partial \lambda}{\partial x}\right)^{2}+\frac{d u}{d \lambda} \frac{\partial^{2} \lambda}{\partial x^{2}}\right\}\right] \text {. } \\
& \frac{\partial \tilde{u}}{\partial Z}=\frac{8(1-2 \eta) A x}{a^{3}}\left\{\operatorname{sn}^{2} u\right\} \frac{d u}{d \lambda} \frac{\partial \lambda}{\partial Z} \\
& +\frac{8 A Z}{a b^{2}}\left[\left\{2 k^{\prime 2} \frac{\operatorname{sn}^{2} u}{\operatorname{cn}^{2} u}\right\} \frac{d u}{d \lambda} \frac{\partial \lambda}{\partial x}\right. \\
& +Z\left\{2 k^{\prime 2} \frac{\operatorname{sn} u \operatorname{dn} u}{\mathrm{cn}^{3} u}\right\}\left(\frac{d u}{d \lambda}\right)^{2} \frac{\partial \lambda}{\partial x} \frac{\partial \lambda}{\partial Z} \\
& \left.+Z\left\{-k^{\prime 2} \frac{\mathrm{sn}^{2} u}{\operatorname{cn}^{2} u}\right\}\left\{\frac{d^{2} u}{d \lambda^{2}} \frac{\partial \lambda}{\partial x} \frac{\partial \lambda}{\partial Z}+\frac{d u}{d \lambda} \frac{\partial^{2} \lambda}{\partial x \partial Z}\right\}\right] \\
& \frac{\partial v}{\partial x}=\frac{8(1-2 \eta) A y}{a^{3}}\left\{\frac{\operatorname{sn}^{2} u}{\mathrm{dn}^{2} u}\right\} \frac{d u}{d \lambda} \frac{\partial \lambda}{\partial x} \\
& +\frac{8 A Z^{2}}{a b^{2}}\left[\left\{2 k^{\prime 2} \frac{\operatorname{sn} u \operatorname{dn} u}{\operatorname{cn}^{3} u}\right\}\left(\frac{d u}{d \lambda}\right)^{2} \frac{\partial \lambda}{\partial y} \frac{\partial \lambda}{\partial x}\right. \\
& \left.+\left\{k^{\prime 2} \frac{\mathrm{sn}^{2} u}{\operatorname{cn}^{2} u}\right\}\left\{\frac{d^{2} u}{d \lambda^{2}} \frac{\partial \lambda}{\partial y} \frac{\partial \lambda}{\partial x}+\frac{d u}{d \lambda} \frac{\partial^{2} \lambda}{\partial y \partial x}\right\}\right]
\end{aligned}
$$




$$
\begin{aligned}
& \frac{\partial v}{\partial y}=\frac{8(1-2 \eta) A}{a^{3} k^{2} k^{\prime 2}}\left\{E(u)-k^{\prime 2} u-\frac{k^{2} \operatorname{sn} u \operatorname{cn} u}{\operatorname{dn} u}\right\} \frac{d u}{d \lambda} \frac{\partial \lambda}{\partial Z} \\
& +\frac{8(1-2 \eta) A y}{a^{3}}\left\{\frac{\operatorname{sn}^{2} u}{\operatorname{dn}^{2} u}\right\} \frac{d u}{d \lambda} \frac{\partial \lambda}{\partial y} \\
& +\frac{8 A Z^{2}}{a b^{2}}\left[\left\{2 k^{\prime 2} \frac{\operatorname{sn} u \operatorname{dn} u}{\operatorname{cn}^{3} u}\right\}\left(\frac{d u}{d \lambda}\right)^{2}\left(\frac{\partial \lambda}{\partial y}\right)^{2}\right. \\
& +\left\{k^{\prime 2} \frac{\mathrm{sn}^{2} u}{\operatorname{cn}^{2} u}\left\{\frac{d^{2} u}{d \lambda^{2}}\left(\frac{\partial \lambda}{\partial y}\right)^{2}+\frac{d u}{d \lambda} \frac{\partial^{2} \lambda}{\partial y^{2}}\right\}\right] \\
& \frac{\partial v}{\partial Z}=\frac{8(1-2 \eta) A y}{a^{3}}\left\{\frac{\operatorname{sn}^{2} u}{\mathrm{dn}^{2} u}\right\} \frac{d u}{d \lambda} \frac{\partial \lambda}{\partial Z} \\
& +\frac{8 A Z}{a b^{2}}\left[\left\{2 k^{\prime 2} \frac{\mathrm{sn}^{2} u}{\mathrm{cn}^{2} u}\right\} \frac{d u}{d \lambda} \frac{\partial \lambda}{\partial y}\right. \\
& +Z\left\{2 k^{2} \frac{\operatorname{sn} u \operatorname{dn} u}{\mathrm{cn}^{3} u}\right\}\left(\frac{d u}{d \lambda}\right)^{2} \frac{\partial \lambda}{\partial y} \frac{\partial \lambda}{\partial Z} \\
& \left.+Z\left\{k^{\prime 2} \frac{\mathrm{sn}^{2} u}{\mathrm{cn}^{2} u}\right\}\left\{\frac{d^{2} u}{d \lambda^{2}} \frac{\partial \lambda}{\partial y} \frac{\partial \lambda}{\partial Z}+\frac{d u}{d \lambda} \frac{\partial^{2} \lambda}{\partial y \partial Z}\right\}\right] \\
& \frac{\partial w}{\partial x}=\frac{8 A Z}{a b^{2}}\left[-(1-2 v)\left\{k^{\prime 2} \frac{\mathrm{sn}^{2} u}{\mathrm{cn}^{2} u}\right\} \frac{d u}{d \lambda} \frac{\partial \lambda}{\partial x}\right. \\
& +Z\left\{2 k^{\prime 2} \frac{\operatorname{sn} u \operatorname{dn} u}{\operatorname{cn}^{3} u}\right\}\left(\frac{d u}{d \lambda}\right)^{2} \frac{\partial \lambda}{\partial x} \frac{\partial \lambda}{\partial Z} \\
& \left.+Z\left\{k^{\prime 2} \frac{\operatorname{sn}^{2} u}{\operatorname{cn}^{2} u}\right\}\left\{\frac{d^{2} u}{d \lambda^{2}} \frac{\partial \lambda}{\partial x} \frac{\partial \lambda}{\partial Z}+\frac{d u}{d \lambda} \frac{\partial^{2} \lambda}{\partial x \partial Z}\right\}\right] \\
& \frac{\partial w}{\partial y}=\frac{8 A Z}{a b^{2}}\left[-(1-2 v)\left\{k^{\prime 2} \frac{\mathrm{sn}^{2} u}{\mathrm{cn}^{2} u}\right\} \frac{d u}{d \lambda} \frac{\partial \lambda}{\partial y}\right. \\
& +Z\left\{2 k^{\prime 2} \frac{\operatorname{sn} u \operatorname{dn} u}{\operatorname{cn}^{2} u}\right\}\left(\frac{d u}{d \lambda}\right)^{2} \frac{\partial \lambda}{\partial Z} \frac{\partial \lambda}{\partial y} \\
& \left.+Z\left\{k^{\prime 2} \frac{\mathrm{sn}^{2} u}{\mathrm{cn}^{2} u}\right\}\left\{\frac{d^{2} u}{d \lambda^{2}} \frac{\partial \lambda}{\partial Z} \frac{\partial \lambda}{\partial y}+\frac{d u}{d \lambda} \frac{\partial^{2} \lambda}{\partial Z \partial y}\right\}\right]
\end{aligned}
$$


To calculate these derivatives, some additional partial derivatives of $\lambda$ need to be calculated. These are given by

$$
\begin{gathered}
\frac{\partial^{2} \lambda}{\partial x \partial Z}=\frac{x Z}{4 h_{1}^{4} \lambda\left(a^{2}+\lambda\right)}\left[-\frac{1}{\lambda}-\frac{1}{a^{2}+\lambda}+\frac{1}{2 h_{1}^{2}}\left\{\frac{x^{2}}{\left(a^{2}+\lambda\right)^{3}}+\frac{y^{2}}{\left(b^{2}+\lambda\right)^{3}}+\frac{Z^{2}}{\lambda^{2}}\right\}\right], \\
\frac{\partial^{2} \lambda}{\partial x^{2}}=\frac{1}{2\left(a^{2}+\lambda\right) h_{1}^{2}}\left[1-\frac{x^{2}}{\left(a^{2}+\lambda\right)^{2} h_{1}^{2}}+\frac{x^{2}}{4\left(a^{2}+\lambda\right) h_{1}^{4}}\left\{\frac{x^{2}}{\left(a^{2}+\lambda\right)^{3}}+\frac{y^{2}}{\left(b^{2}+\lambda\right)^{3}}+\frac{Z^{2}}{\lambda^{2}}\right\}\right], \\
\frac{\partial^{2} \lambda}{\partial y^{2}}=\frac{1}{2\left(b^{2}+\lambda\right) h_{1}^{2}}\left[1-\frac{y^{2}}{\left(b^{2}+\lambda\right)^{2} h_{1}^{2}}+\frac{y^{2}}{4\left(b^{2}+\lambda\right) h_{1}^{4}}\left\{\frac{x^{2}}{\left(a^{2}+\lambda\right)^{3}}+\frac{y^{2}}{\left(b^{2}+\lambda\right)^{3}}+\frac{Z^{2}}{\lambda^{2}}\right\}\right],
\end{gathered}
$$

and

$$
\frac{\partial^{2} \lambda}{\partial Z^{2}}=\frac{1}{2 \lambda h_{1}^{2}}\left[1-\frac{Z^{2}}{\lambda^{2} h_{1}^{2}}+\frac{Z^{2}}{4 \lambda h_{1}^{4}}\left\{\frac{x^{2}}{\left(a^{2}+\lambda\right)^{3}}+\frac{y^{2}}{\left(b^{2}+\lambda\right)^{3}}+\frac{Z^{2}}{\lambda^{2}}\right\}\right]
$$

For vertical fractures that are taller than they are long, the normal tilt is given by interchanging $\mathrm{a}$ and $\mathrm{b}$ (essentially switching the length and the height) and using $\partial w / \partial x$. The parallel tilt is given by $\partial v / \partial x$.

For fractures with dip, it is necessary to rotate the displacement gradients into the correct orientation and it is necessary to find the correct spatial parameters. Considering the transformation first, Figure 4 again shows the fracture with dip and the observation well and then the rotated view of this geometry. As in the $2 \mathrm{D}$ case, it is again observed that the two displacements of interest, the one normal to the fracture $\left(u_{n}\right)$ and the one parallel to the fracture $\left(u_{p}\right)$ are given by

$$
\begin{gathered}
u_{n}=w \cos \gamma+v \sin \gamma \\
u_{p}=\tilde{u}
\end{gathered}
$$


where $\gamma$ is the angle of the fracture plane references to the vertical (e.g., zero is a vertical fracture). The tiltmeter array measures the variation of these displacement derivatives along the s direction,

$$
\frac{\partial u_{n}}{\partial s}=\frac{\partial u_{n}}{\partial Z} \frac{d Z}{d s}+\frac{\partial u_{n}}{\partial y} \frac{d y}{d s}
$$

and

$$
\frac{\partial u_{p}}{\partial s}=\frac{\partial u_{p}}{\partial Z} \frac{d Z}{d s}+\frac{\partial u_{p}}{\partial y} \frac{d y}{d s}
$$

Since the two spatial derivatives are given by

$$
\frac{d Z}{d s}=-\sin \gamma \text { and } \frac{d y}{d s}=\cos \gamma
$$

the tilt derivatives can be reduced to

$$
\frac{\partial u_{n}}{\partial s}=\sin \gamma \cos \gamma\left[-\frac{\partial w}{\partial Z}+\frac{\partial v}{\partial y}\right]-\sin ^{2} \gamma \frac{\partial v}{\partial Z}+\cos ^{2} \lambda \frac{\partial w}{\partial y}
$$

and

$$
\frac{\partial u_{p}}{\partial s}=-\sin \gamma \frac{\partial \tilde{u}}{\partial Z}+\cos \gamma \frac{\partial \tilde{u}}{\partial y}
$$

If the fracture is taller than it is long, the $a$ and $b$ should be switched and the appropriate derivatives are

$$
\frac{\partial u_{n}}{\partial s}=\sin \gamma \cos \gamma\left[-\frac{\partial w}{\partial Z}+\frac{\partial \tilde{u}}{\partial x}\right]-\sin ^{2} \gamma \frac{\partial \tilde{u}}{\partial Z}+\cos ^{2} \gamma \frac{\partial w}{\partial x}
$$

and

$$
\frac{\partial u_{p}}{\partial s}=-\sin \gamma \frac{\partial v}{\partial Z}+\cos \gamma \frac{\partial v}{\partial x}
$$


The final task is to determine the correct geometric parameters to characterize the distance from the fracture to the tiltmeters. Referring to the unrotated schematic in Figure 6, it can be found from geometry considerations that

$$
\begin{gathered}
\hat{x}=x \\
\hat{y}=Z_{o} \sin \gamma+y_{o} \cos \gamma
\end{gathered}
$$

and

$$
\hat{Z}=Z_{o} \cos \gamma-y_{o} \sin \gamma
$$

where the variables with hats are the correct distances to use in the analysis. Of course, if the fracture is taller than it is long, then the $\mathrm{x}$ and $\mathrm{y}$ variable need to be reversed so that

$$
\begin{gathered}
\hat{x}=Z_{o} \sin \gamma+x_{o} \cos \gamma \\
\hat{y}=y,
\end{gathered}
$$

where the vertical distance from the crack centerline to the tiltmeter of interest is now $x_{o}$. These equations complete the analysis of downhole tilt data for any elliptic fracture of any geometry, as long as it is far from the free surface.

\subsection{D Fracture With Internal Pressure Distribution}

England and Green [1963] have solved the problem of a 2D fracture with variable pressure distribution in the crack, which is equivalent to the case of constant internal pressure with an arbitrary number of layers having variable stress, as shown in Figure 6. This problem is of interest when the crack is large and crosses into several layers with different stresses. Since higher stress layers will clamp the fracture width and reduce the magnitude of the tilts, analyses that use a homogeneous medium could be significantly in error. However, this solution can only be used for a fracture that is normal to the bedding (e.g., a vertical fracture with horizontal beds). 


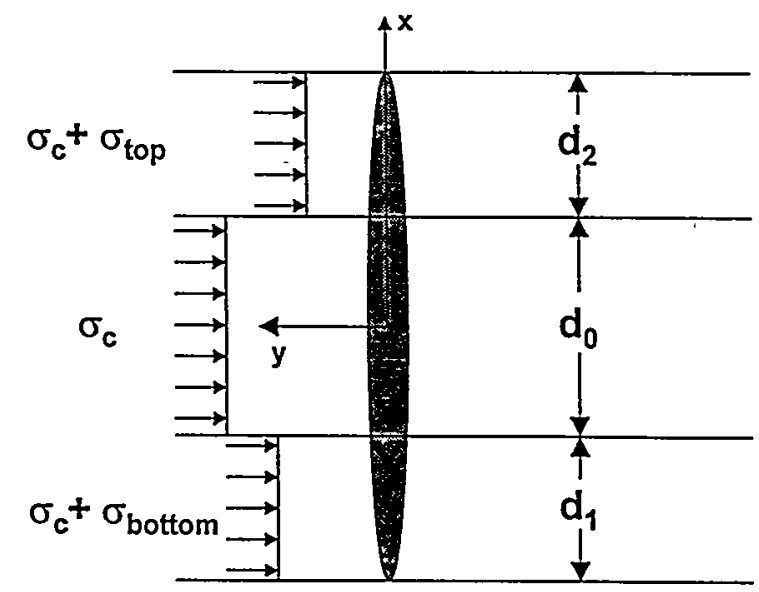

Figure 6. Geometry for crack with layered stress distribution.

As with the previous $2 \mathrm{D}$ solution, there is only one tilt component developed by such a crack, the one normal to the fracture plane. The solution to the problem follows the development of crack problems by Green and Zerna [1968] in which the displacements and their derivative with respect to $\mathrm{x}$ are

$$
D=u_{x}+i u_{y}=\frac{2(1+v)}{E}\left\{(3-4 v) \Omega(z)-\Omega^{\prime}(\bar{z})+(\bar{z}-z) \Omega^{\prime}(\bar{z})\right\}
$$

and

$$
\frac{\partial D}{\partial x}=\frac{2(1+v)}{E}\left\{(3-4 v) \Omega^{\prime}(z)-\Omega^{\prime}(\bar{z})+(\bar{z}-z) \Omega^{n}(\bar{z})\right\}
$$

The England and Green [1963] solution for the function $\Omega$ is of the form

$$
\Omega(z)=\int \frac{F(t)+z G(t)}{\sqrt{z^{2}-t^{2}}} d t
$$

where the functions $F(t)$ and $G(t)$ are given by

$$
F(t)=-\frac{t}{2 \pi} \int \frac{f(u) d u}{\sqrt{t^{2}-u^{2}}}
$$

and 


$$
G(t)=-\frac{1}{2 \pi t} \int \frac{u g(u) d u}{\sqrt{t^{2}-u^{2}}}
$$

where $f(u)$ and $g(u)$ are even and odd functions, respectively, that make up the internal pressure, $p(u)$, in the fracture,

$$
p(u)=f(u)+g(u)
$$

For example, a constant pressure, $\mathrm{P}$, would result in $f(u)=\mathrm{P}$ and $g(u)=0$. A pressure of $\mathrm{P}_{1}$ on the top half of the fracture and $\mathrm{P}_{2}$ on the bottom half would result in $\mathrm{f}(\mathrm{u})=\left(\mathrm{P}_{1}+\mathrm{P}_{2}\right) / 2$ and $g(u)=\left(P_{1}-P_{2}\right) / 2$, so that $g(u)=\left(P_{1}-P_{2}\right) / 2$ for $x>0$ and $g(u)=-\left(P_{1}-P_{2}\right) / 2$ for $x<0$.

Using the imaginary part of the $\mathrm{x}$ derivative of the displacement function, $\mathrm{D}$, and taking the necessary derivatives, the iriclination of a downhole tiltmeter can be found as

$$
\frac{\partial u_{y}}{\partial x}=\frac{2(1+v)}{E} \int_{0}\left[-4(1-v)\left\{\frac{A_{1}}{\xi}+\frac{A_{2}}{\xi^{3}}+\frac{A_{3}}{\xi^{3}}\right\}+2 y\left\{\frac{B_{1}}{\xi^{3}}+\frac{B_{2}}{\xi^{3}}\right\}-\left\{\frac{C_{1}}{\xi^{5}}+\frac{C_{2}}{\xi^{5}}\right\}\right]
$$

where

$$
\begin{aligned}
& A_{1}=G(t) \sin \eta \\
& A_{2}=F(t)[y \cos (3 \eta)-x \sin (3 \eta)] \\
& A_{3}=G(t)\left[2 x y \cos (3 \eta)-\left(x^{2}-y^{2}\right) \sin (3 \eta)\right] \\
& B_{1}=F(t) \cos (3 \eta) \\
& B_{2}=3 G(t)[x \cos (3 \eta)+y \sin (3 n)] \\
& C_{1}=3 F(t)\left[\left(x^{2}-y^{2}\right) \cos (5 \eta)+2 x y \sin (5 \eta)\right] \\
& C_{2}=3 G(t)\left[\left(x^{3}-3 x y^{2}\right) \cos (5 \eta)-\left(y^{3}-3 x^{2} y\right) \sin (5 \eta)\right]
\end{aligned}
$$

and 


$$
\begin{aligned}
& \xi=\left[\left(x^{2}+y^{2}+t^{2}\right)^{2}+4 x^{2} y^{2}\right]^{1 / 4} \\
& \eta=\frac{1}{2} \tan ^{-1}\left[\frac{2 x y}{x^{2}+y^{2}+t^{2}}\right]
\end{aligned}
$$

Integration of the tilt equation is handled numerically. To get physically reasonable tilts, the pressure must be large enough to support a fracture of the specified height. In actual practice, this tilt calculation is combined with the corresponding calculation of the fracture height and width for a given internal pressure and layered-stress medium [Warpinski, 1989]. This calculation assures that the fracture does not close back on itself.

\subsection{Model Results}

The models can be used to help understand how the tilt field is affected by the important parameters and to determine optimum locations for downhole tilt monitoring. Each model may be more useful for examining a specific parameter, so each of the four models is used here in some capacity. For all cases studied here, Young's modulus has been kept at $27.6 \mathrm{GPa}$ and Poisson's ratio is 0.2 . The internal fracture pressure is usually $6.89 \mathrm{Mpa}$ and most other parameters have been varied.

The 2D model is very useful for examining the effect of fracture height and monitoring distance on the amplitude decay and tilt radiation. The effect of fracture height at a prescribed monitoring distance $(152 \mathrm{~m})$ is shown in Figures 7 and 8 . Figure 7 shows crack heights of 15.2, 30.5, 61 and $122 \mathrm{~m}$ while Figure 8 has an expanded scale to show 7.6, 15.2, and $30.4 \mathrm{~m}$ cracks. In all cases, the tilt distribution follows the classic S-shaped curve observed in field data [e.g., Branagan et al., 1996; Warpinski et al., 1997a \& 1997b; Wright, 1998; Wright et al., 1998]. The obvious effect of crack height is on the amplitude of the tilt field, which ranges from less than a microradian for the small fracture heights to as much as 50 microradians for the $122 \mathrm{~m}$ height 
fracture. Compared to surface tilts that usually are a few tens to a few hundreds of nanoradians, the response of a downhole tiltmeter can be extremely large. Nevertheless, the smaller fractures will be difficult to image if the tiltmeters are not well coupled to the formation or if wellbore or electronic noise is large.

One important observation that can be made of these comparisons concerns the locations of the peak tilt values as a function of fracture height. For large fracture heights relative to the distance to the tiltmeters, the locations of the amplitude peaks are a clear function of the fracture height. However, for small fracture heights as in Figure 8, the locations of the peaks are only slightly changed with fracture height. This behavior shows that there is a limit to how far away the tiltmeters can be to image the fracture accurately and that limit depends on the height of the fracture.

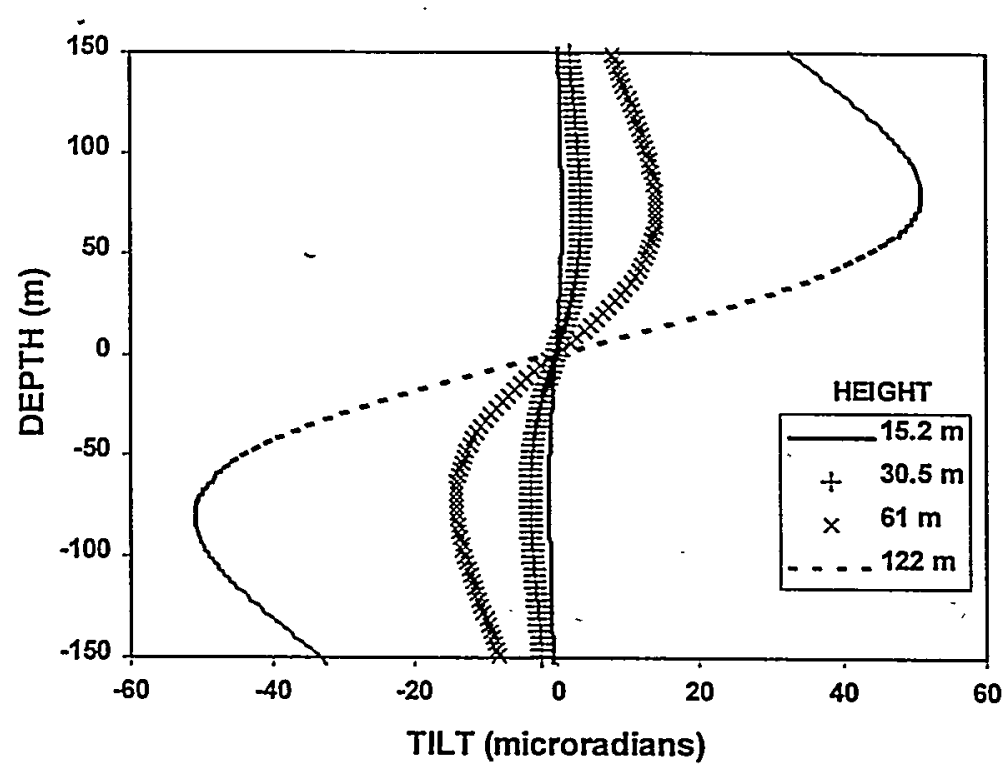

Figure 7. Effect of fracture height at $152 \mathrm{~m}$ monitoring distance, 2D crack. 


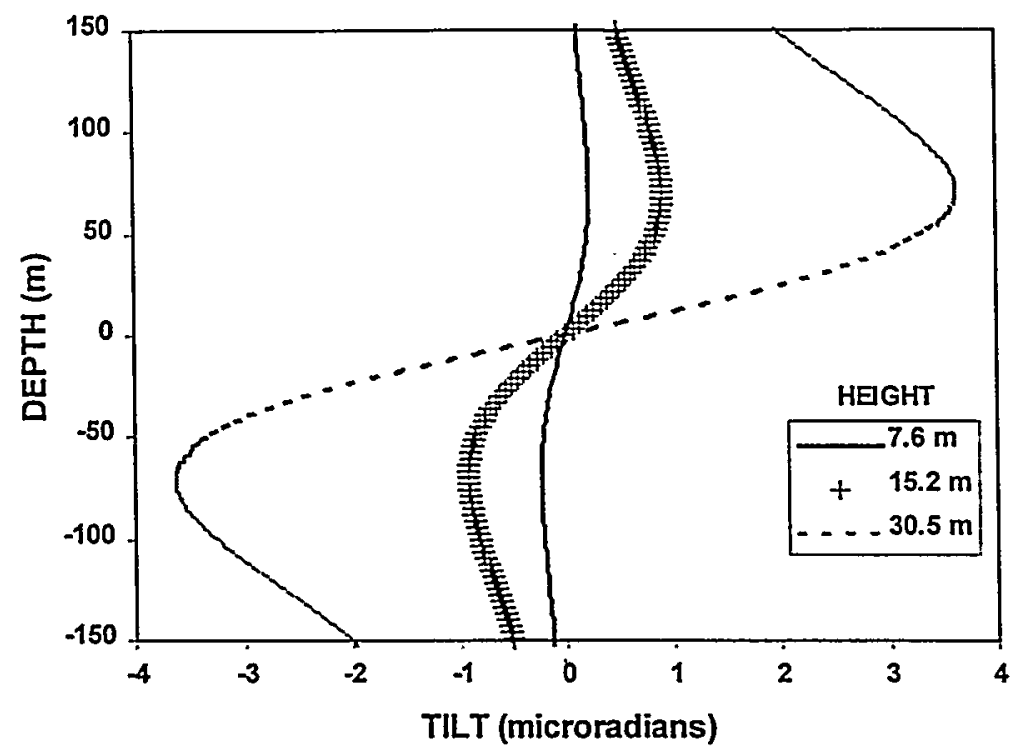

Figure 8. Effect of crack height at $152 \mathrm{~m}$ monitoring distance, 2D crack.

The effect of monitoring distance for a given fracture height is shown in Figure 9 for short monitoring distances and in Figure 10 for greater distances. When the monitoring distance is on the order of the crack height, the peaks in the tilt distribution are nearly aligned with the top and bottom of the fracture. However, for greater monitoring distances the peak locations quickly spread out and cannot be used as a reliable indicator of fracture height. The data in Figure 10 show that the tilts can be measured a long distance from a large fracture, but that the deformation exhibits considerable vertical expansion with monitoring distance and an extremely large tilt array would be required. Assuming that a $300 \mathrm{~m}$ long tilt array is a reasonable maximum for an array, these results suggests that quantitative tilt measurements can be made for distance-toheight ratios of approximately 7-10 for a long fracture (e.g., 2D case). 


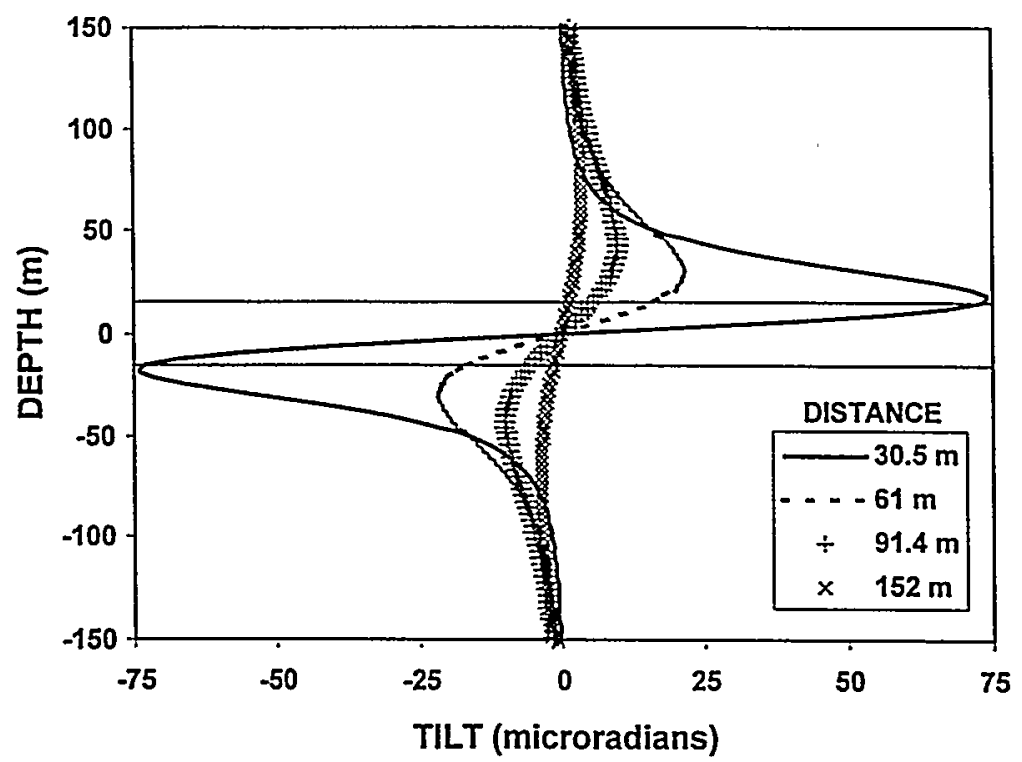

Figure 9. Effect of monitoring distance for a $30.5 \mathrm{~m}$ height fracture, 2D case.

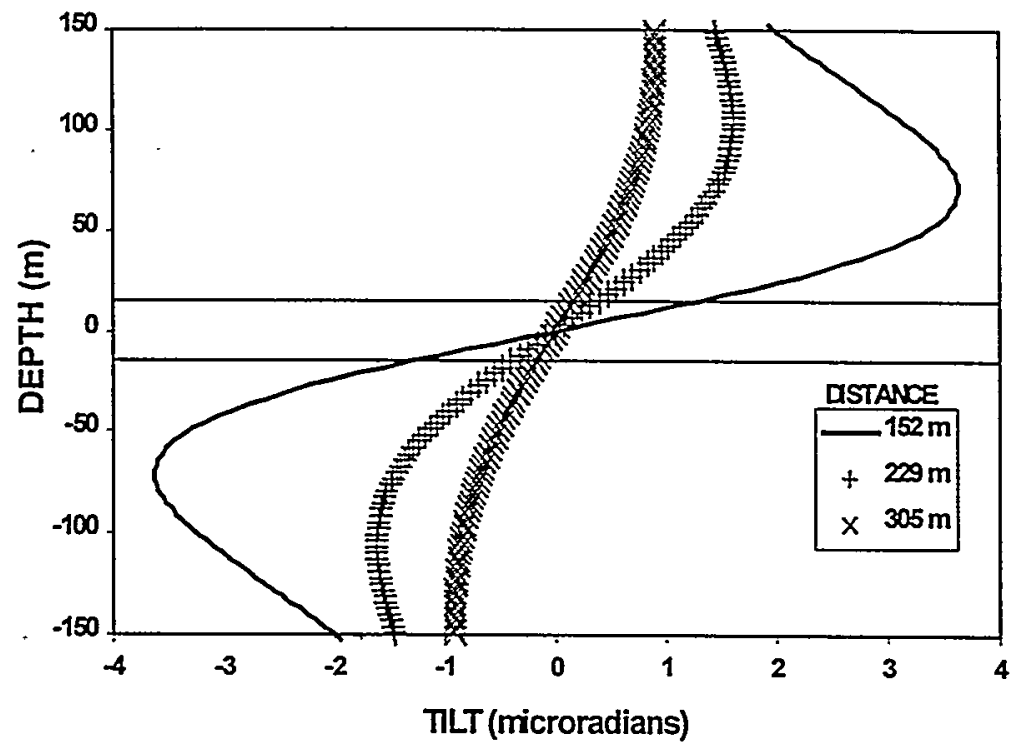

Figure 10. Effect of monitoring distance for a $30.5 \mathrm{~m}$ height fracture, $2 \mathrm{D}$ case.

The effect of the fracture dip on the downhole tilt field for a fracture of $30.5 \mathrm{~m}$ height at a monitoring distance of $152 \mathrm{~m}$ is shown in Figure 11. However, the number given in the legend 
box is the deviation of the fracture from vertical, rather than the fracture dip $(0=$ a vertical fracture) since hydraulic fractures are generally vertical. For small deviations from vertical (inclinations), the effect of the inclination is to cause the tilts to increase in magnitude on the side where the fracture approaches the monitoring array and decrease on the other side. In addition, the locations of the peaks are forced outward and are no longer connected to the fracture height in a clear way. For larger inclinations, the double peak becomes essentially a single peak in the center of the array, while a horizontal fracture looks like a vertical fracture of smaller height. Thus, it can be seen that fracture dip can cause some confusing results unless some other independent information about the fracture geometry is available.

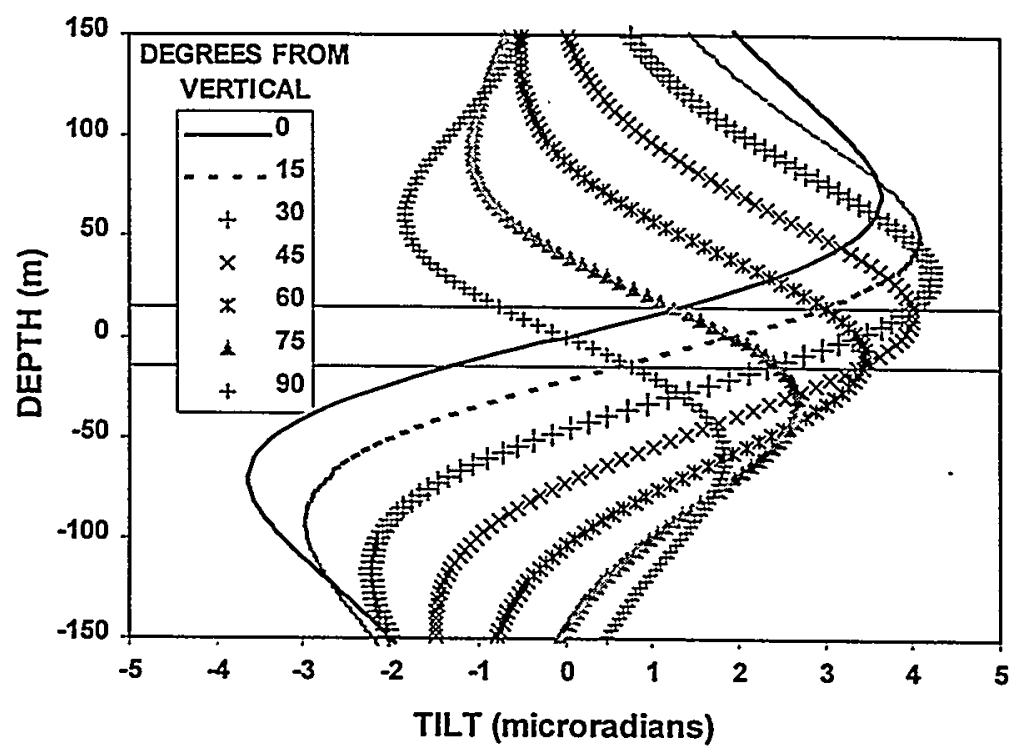

Figure 11. Effect of dip for a $30.5 \mathrm{~m}$ height fracture, $152 \mathrm{~m}$ distant, 2D cased.

The penny-shaped, or radial, fracture model is useful for examining the effects of short fracture lengths on the measured tilt field. Figures 12 and 13 show the ability of a tiltmeter array to measure fractures of various radii from a distance of $152 \mathrm{~m}$, with the downhole tiltmeter array placed directly on the fracture-center orthogonal line (the line orthogonal to the fracture passing 
through the center of the fracture). At this distance, the tiltmeters are able to resolve fractures of greater than $30 \mathrm{~m}$ radius, but may have difficulty with smaller fractures.

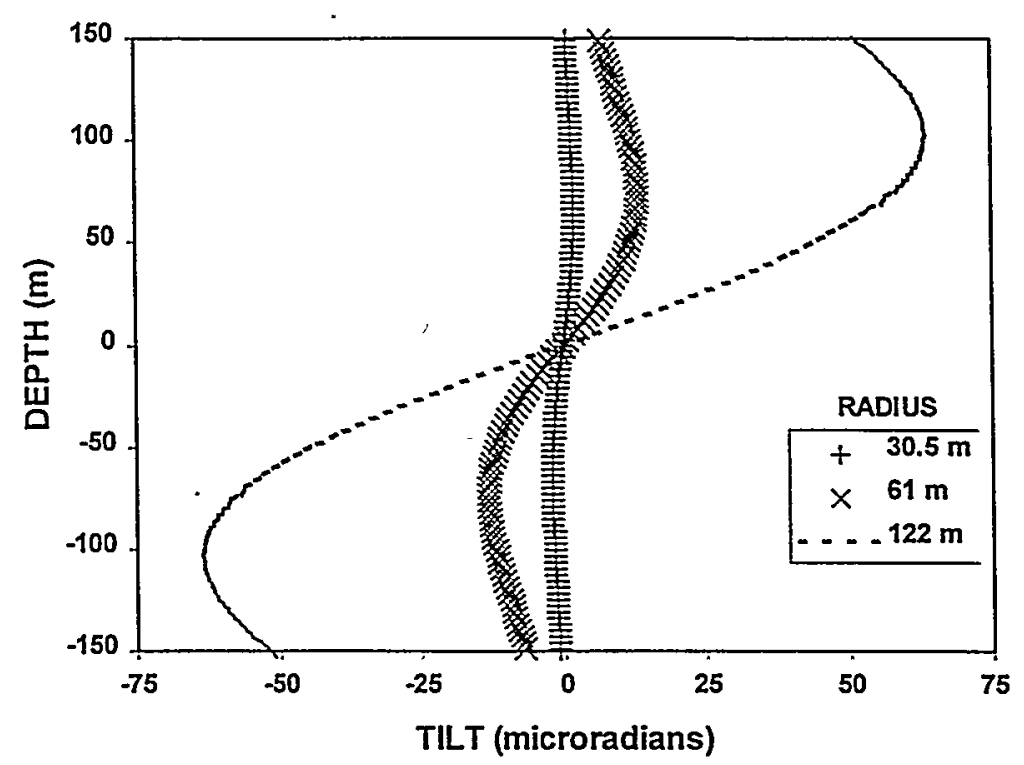

Figure 12. Effect of fracture radius for a monitoring distance of $152 \mathrm{~m}$, penny-shaped fracture.

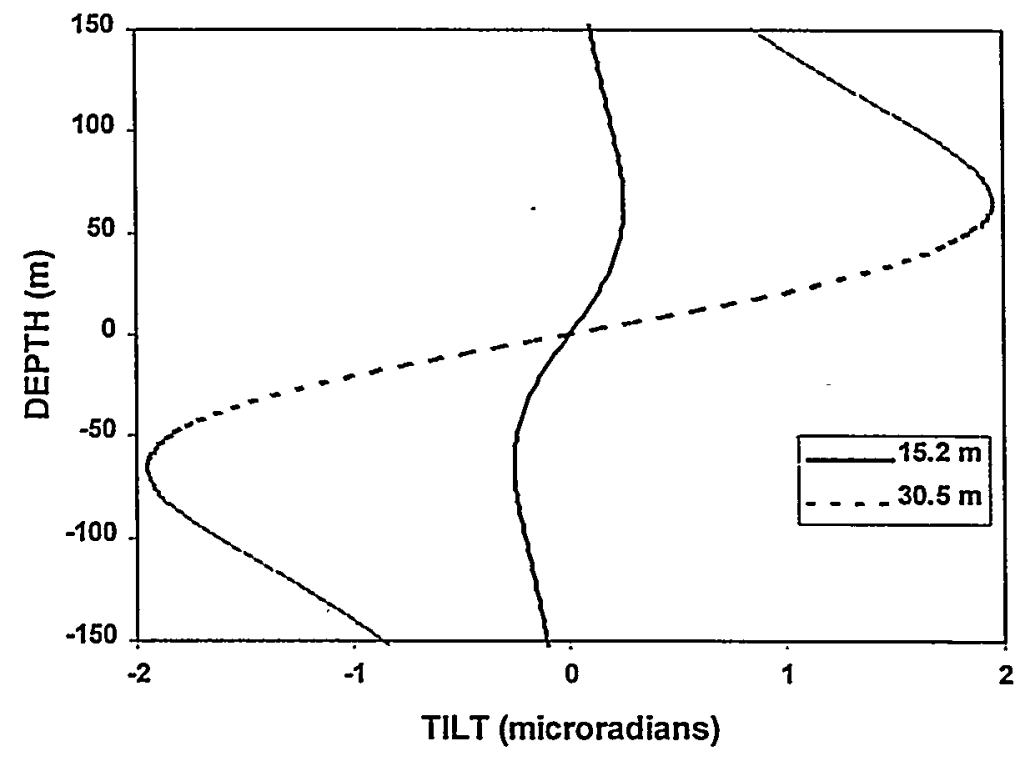

Figure 13. Effect of fracture radius for a monitoring distance of $152 \mathrm{~m}$, penny-shaped fracture. 
Figures 14 and 15 show the effect of monitoring distance on the tilt fields for a $61 \mathrm{~m}$ fracture height. For radial fractures, the location of the peaks does not match well with the top and bottom of the fracture, even for fractures that have a large radius compared to the distance to the monitoring array. As would be expected, the effect of distance on amplitude reduction is greater for the penny-shaped case than it is for the $2 \mathrm{D}$ case

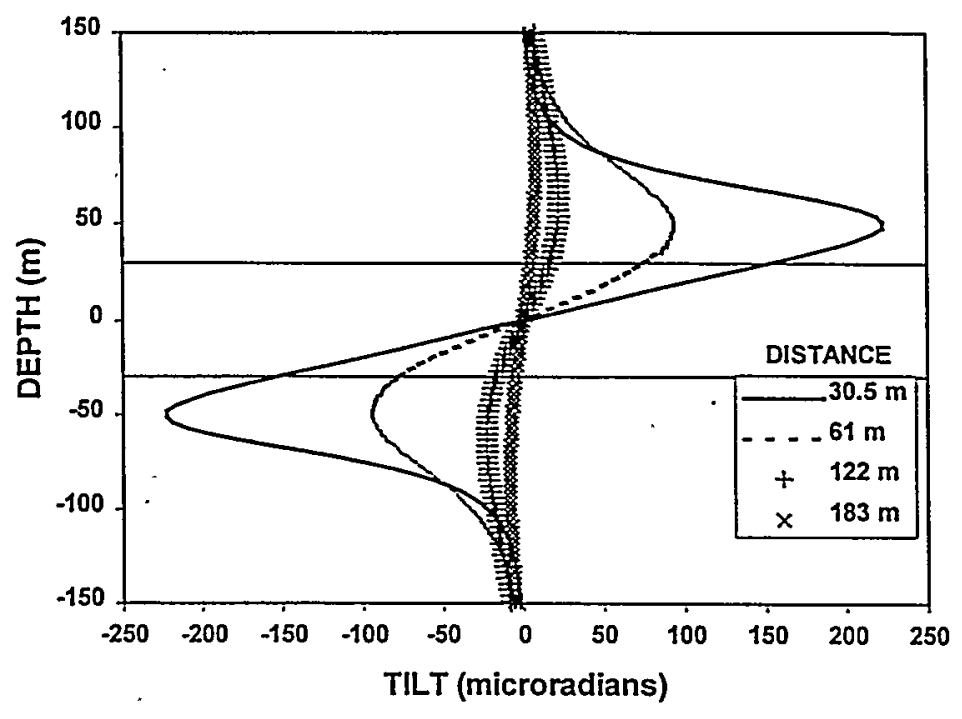

Figure 14. Effect of monitoring distance for a $61 \mathrm{~m}$ radius fracture, penny-shaped case.

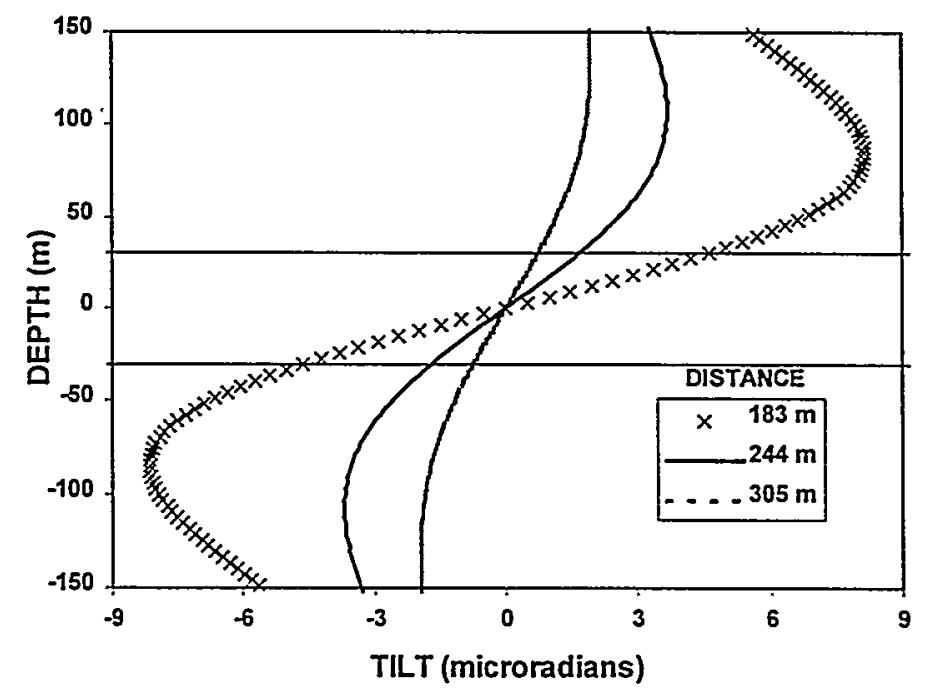

Figure 15. Effect of monitoring distance for a $61 \mathrm{~m}$ radius fracture, penny-shaped case. 
The previous calculations for a penny-shaped crack have been on the centerline of the fracture so that the response is a maximum and there is no parallel tilt. The effect of the offset distance for a $61 \mathrm{~m}$ radius crack at a monitoring distance of $152 \mathrm{~m}$ is shown in Figure 16 for the normal tilt distribution. As can be seen, the normal tilt decreases as the monitoring array is moved farther away from the centerline of the fracture.

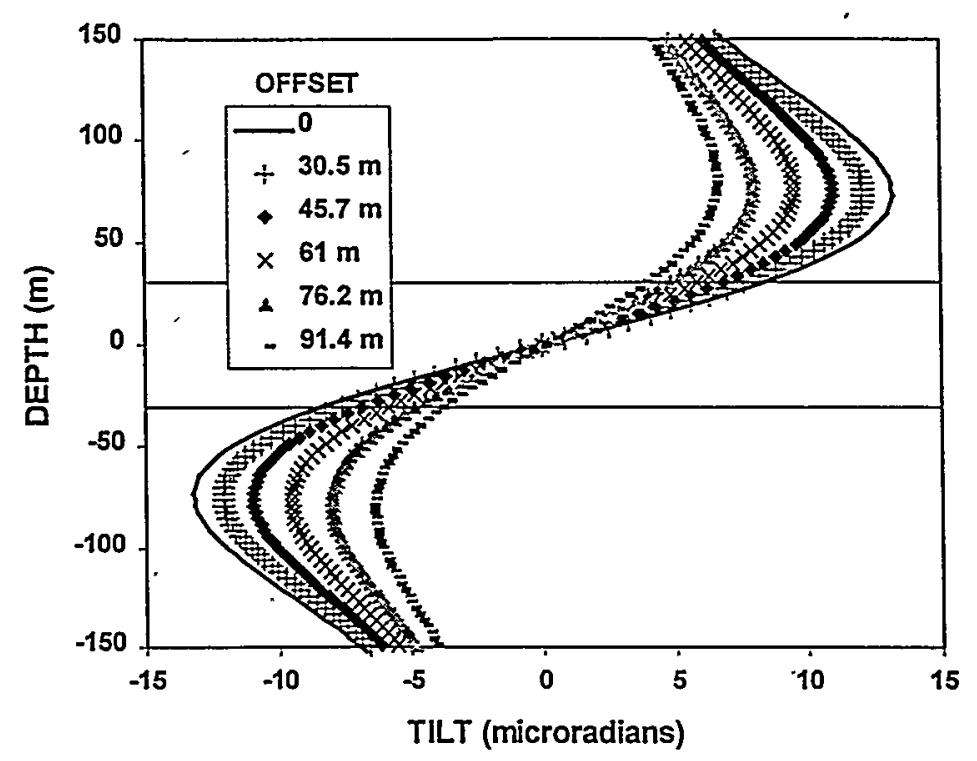

Figure 16. Effect of offset distance on normal tilt distribution for $61 \mathrm{~m}$ radius fracture at $152 \mathrm{~m}$ distance, penny-shape case.

In addition to the changes in the normal tilt, moving the monitoring array to an offset position also induces a tilt field parallel to the crack strike. The parallel tilt distribution for this same case is shown in Figure 17. For no offset, the parallel tilt is zero everywhere due to symmetry. For increasing offset, the parallel tilt increases until it reaches a maximum at a location about 1.25 radii from the center, after which it begins to decrease. 


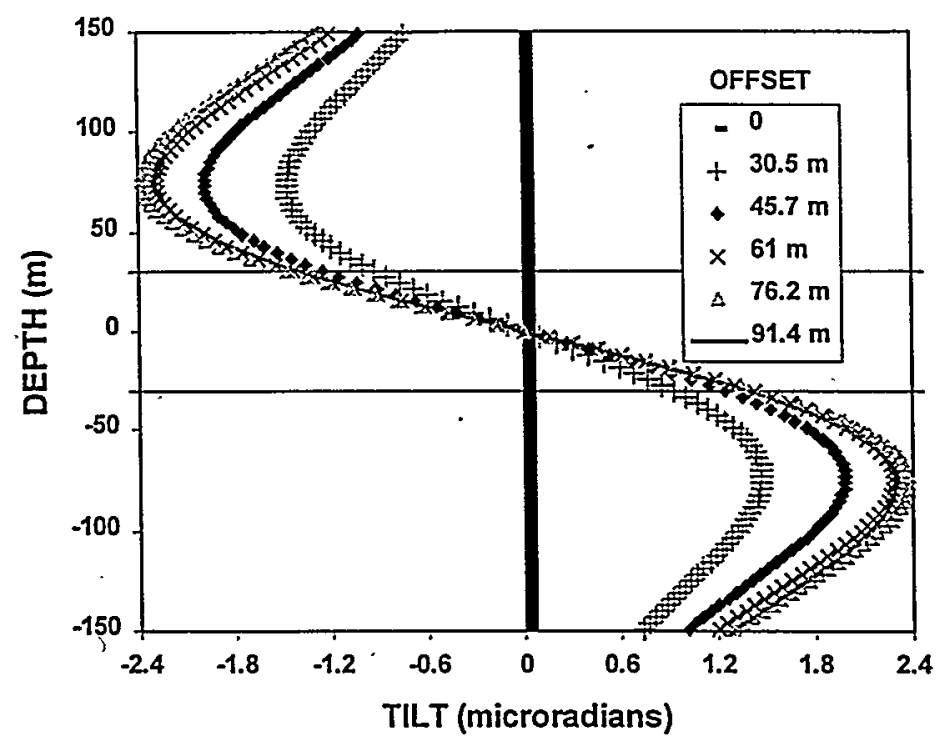

Figure 17. Effect of offset distance on parallel tilt distribution for $61 \mathrm{~m}$ radius fracture at $152 \mathrm{~m}$ distance, penny-shape case.

The total tilt distribution (the vector sum) for this case, is shown in Figure 18. This plot is not much different than Figure 16 because the majority of the tilt is due to the normal tilt component.

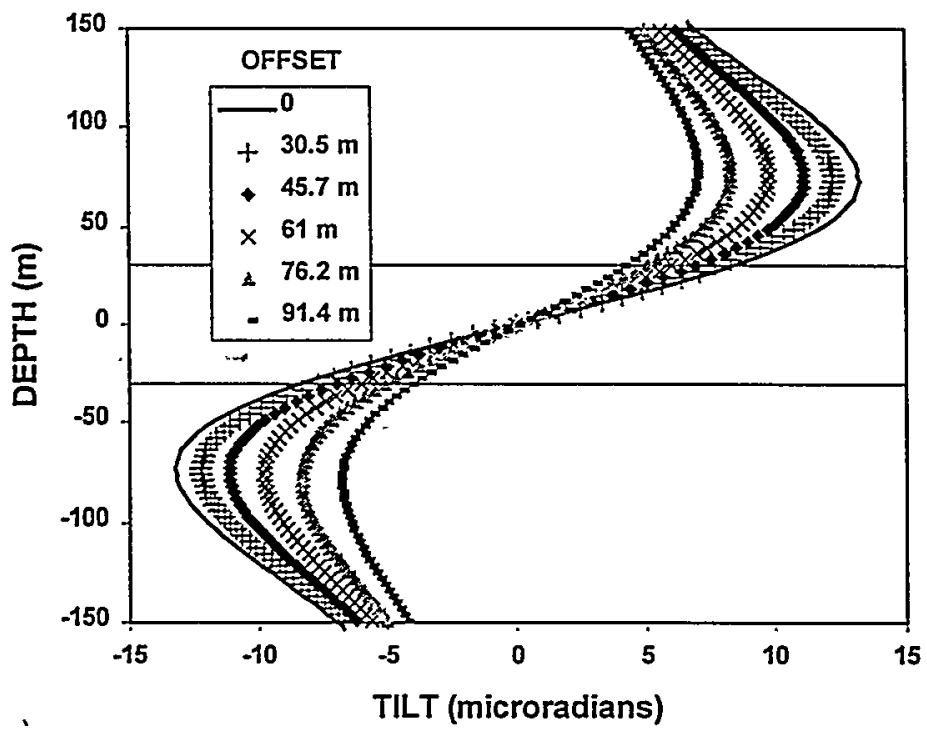

Figure 18. Effect of offset distance on tilt vector-sum distribution for $61 \mathrm{~m}$ radius fracture at $152 \mathrm{~m}$ distance, penny-shape case. 
The elliptic model can be use to assess the effects of fracture length and other parameters. Figure 19 shows the calculated normal tilt distribution for several fracture lengths with the fracture height kept constant at $30.5 \mathrm{~m}$ and the monitoring distance at $152 \mathrm{~m}$. The monitoring array is centered about the fracture so that symmetry causes the parallel component of tilt to be zero. For comparison, the 2D calculation for a $30.5 \mathrm{~m}$ height fracture at a monitoring distance of $152 \mathrm{~m}$ is also shown. The tilt increases considerably as the fracture grows, giving some possibility to extract length as well as height from tiltmeter data. The comparison of the $2 \mathrm{D}$ and $305 \mathrm{~m}$ length cases show that when the ratio of the length to monitoring distance exceeds 2 the 2D calculation provides acceptable accuracy. The $15.2 \mathrm{~m}$ height case is a radial (or pennyshaped) fracture and shows the other limiting case.

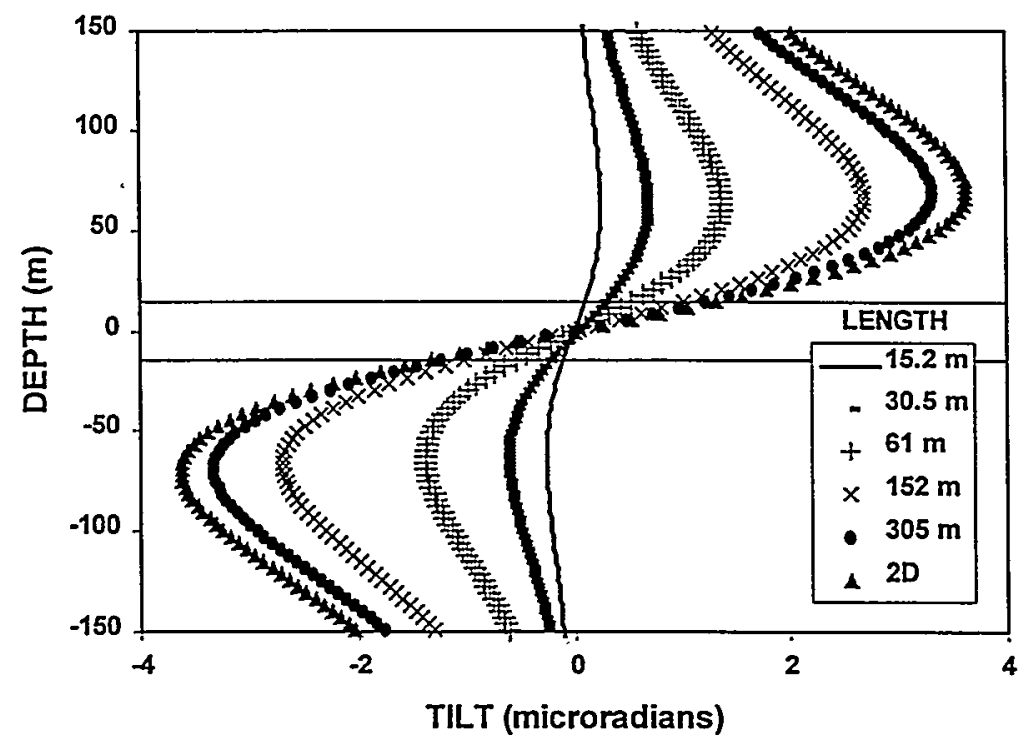

Figure 19. Effect of Fracture Length for a $30.5 \mathrm{~m}$ height fracture at a distance of $152 \mathrm{~m}$.

As would be expected, offsetting the monitoring location away from the centerline results in a reduced normal tilt distribution. Figure 20 shows the normal tilt distribution for a $30.5 \mathrm{~m}$ height fracture at a monitoring distance of $152 \mathrm{~m}$ and an offset of $152 \mathrm{~m}$ for several different crack 
lengths. When the monitoring distance is considerably greater than the fracture height, the effect of offset is difficult to discern from the effect of fracture length (e.g., Figure 19).

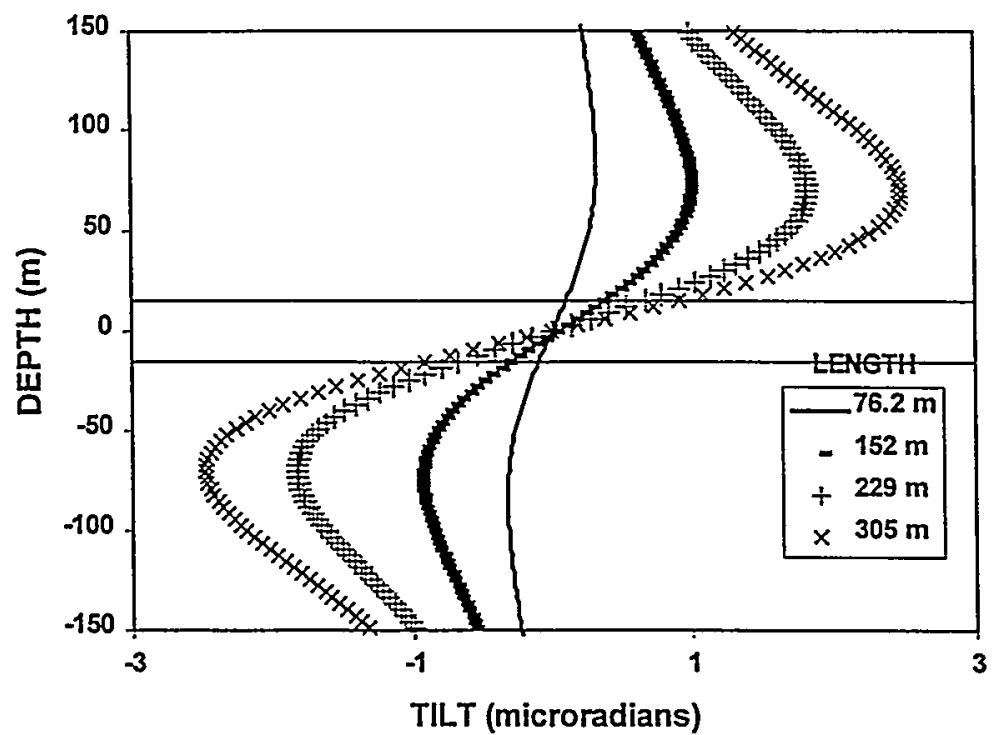

Figure 20. Effect of fracture length on the normal tilt distribution of a $152 \mathrm{~m}$ offset monitoring location for a $30.5 \mathrm{~m}$ height fracture at a monitoring distance of $152 \mathrm{~m}$.

The parallel tilts show a considerably different behavior, with the tilt amplitudes increasing up to a point and then beginning to decrease after the fracture is well past (the parallel tilts must eventually decrease as the fracture approaches a 2D condition with increasing length). 


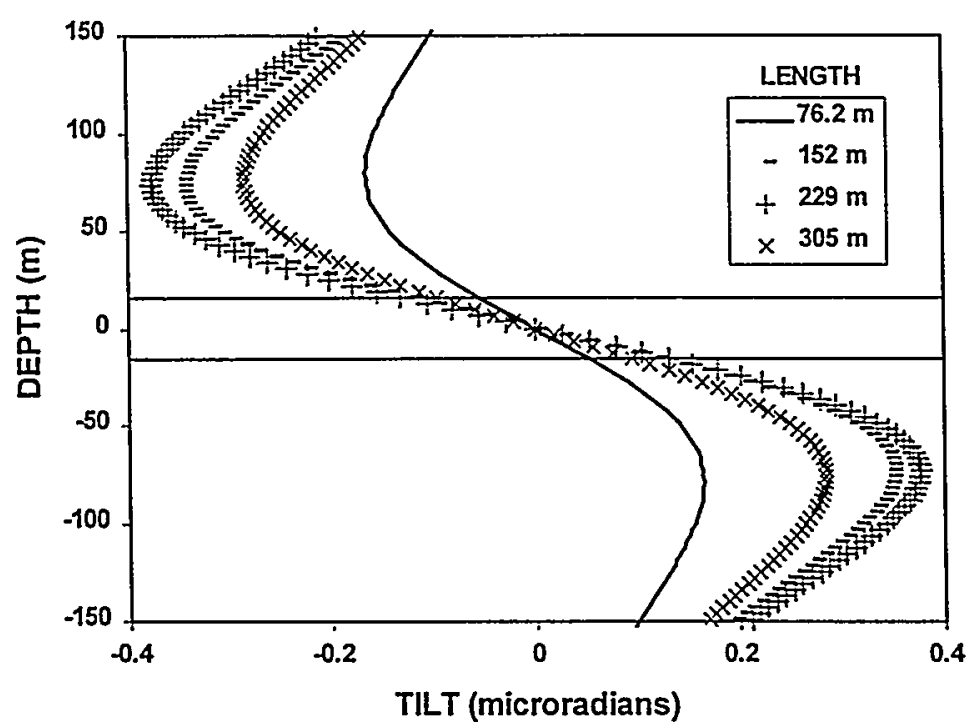

Figure 21. Effect of fracture length on the parallel tilt distribution of a $152 \mathrm{~m}$ offset monitoring location for a $30.5 \mathrm{~m}$ height fracture at a monitoring distance of $152 \mathrm{~m}$.

If the monitoring location is offset from the center, as in the previous case, but is close to the fracture (small monitoring distance), the ability to resolve fracture attributes increases. Figure 22 shows results for a $30.5 \mathrm{~m}$ height fracture of various lengths with a monitoring array offset by $152 \mathrm{~m}$ from the centerline, but a monitoring distance of only $30.5 \mathrm{~m}$. For fractures shorter than the monitoring array offset, the normal tilts are small. As the fracture approaches the monitoring location, the normal tilts increase rapidly. For longer fractures, the normal tilts continue to increase, but at a much slower rate. In addition, being close to the fracture allows for the height of the fracture to be accurately determined by the locations of the peak amplitudes. 


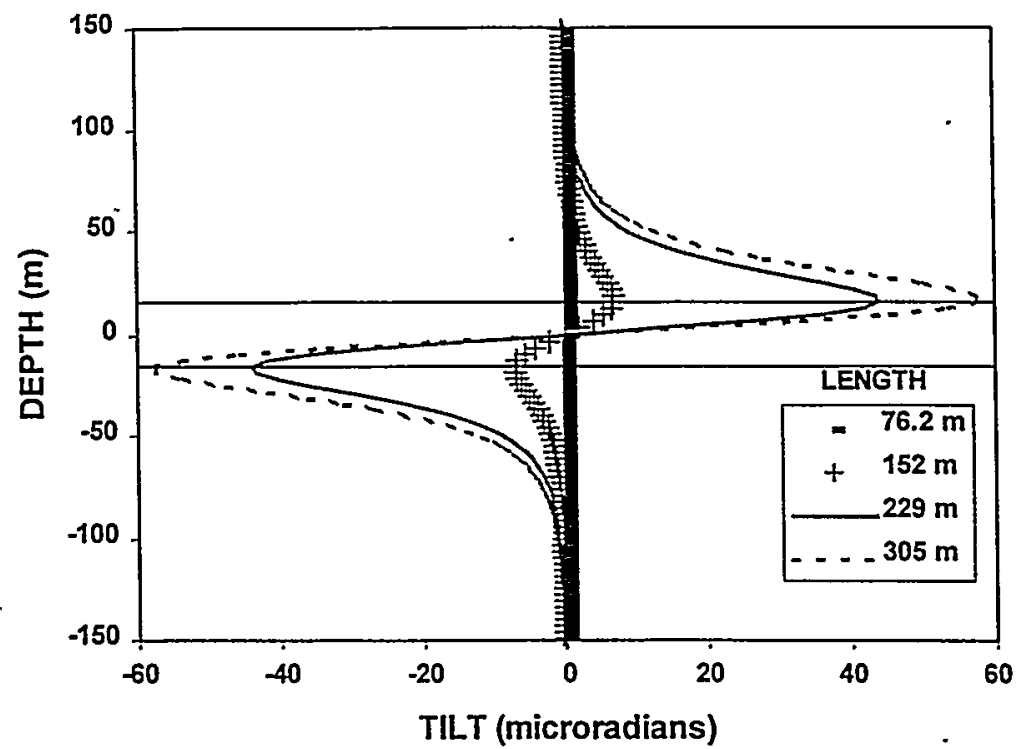

Figure 22. Effect of fracture length on the normal tilt distribution of a $152 \mathrm{~m}$ offset monitoring location for a $30.5 \mathrm{~m}$ height fracture at a monitoring distance of $30.5 \mathrm{~m}$.

The parallel tilt distributions for the same case are shown in Figure 23. The parallel tilts also show a large change as the fracture passes, with increasing amplițde for shorter fractures and decreasing amplitude for larger fractures. The maximum point provides for a very useful calibration point, as the length is known quite accurately at this one point and the height is fixed by the locations of the peaks. Thus the relationship between amplitude and pressure can be accurately determined for later calculations. 


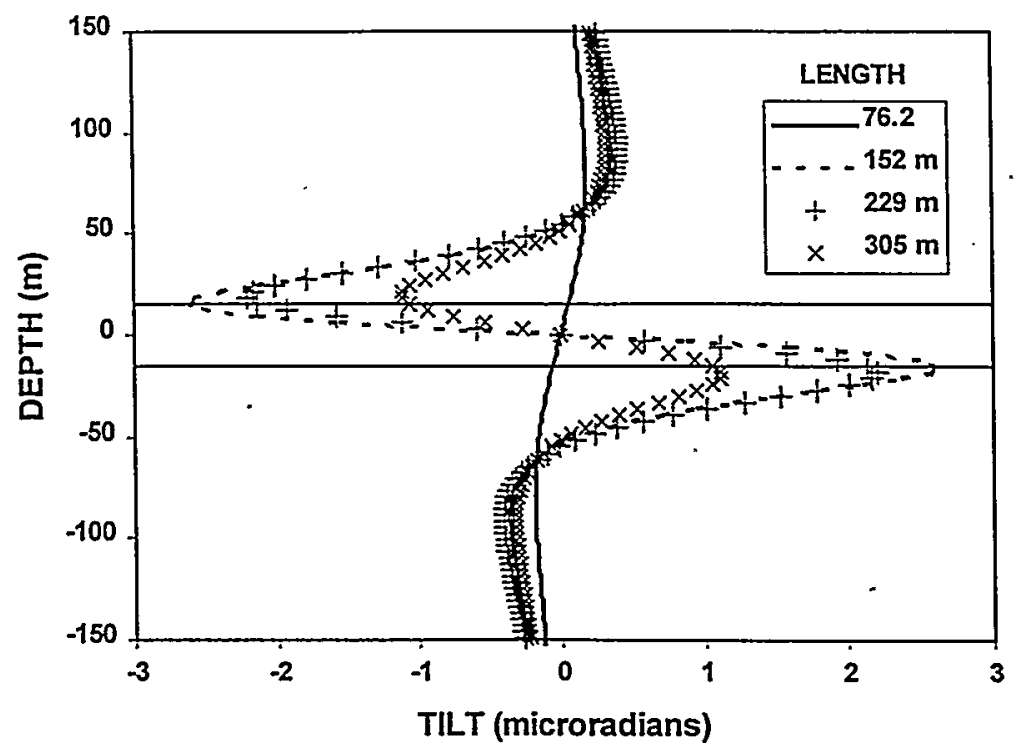

Figure 23. Effect of fracture length on the parallel tilt distribution of a $152 \mathrm{~m}$ offset monitoring location for a $30.5 \mathrm{~m}$ height fracture at a monitoring distance of $30.5 \mathrm{~m}$.

The effect of fracture azimuth on the tilt distribution can also be investigated using the elliptic model. Figure 24 shows the geometry of a fracture at some angle, $\alpha_{2}$ with respect to the line normal to the treatment and monitoring wells. For a zero degree azimuth, the monitoring distance, $z_{m}$ is equal to the distance between the monitoring well and the treatment well, $z_{m o}$ and the offset, $x_{o f f}$ is zero. As the azimuth deviates from 0 , the monitoring distance decreases as

$$
z_{m}=z_{m o} \cos \alpha
$$

and the offset distance increases as

$$
x_{o f f}=z_{m o} \sin \alpha
$$




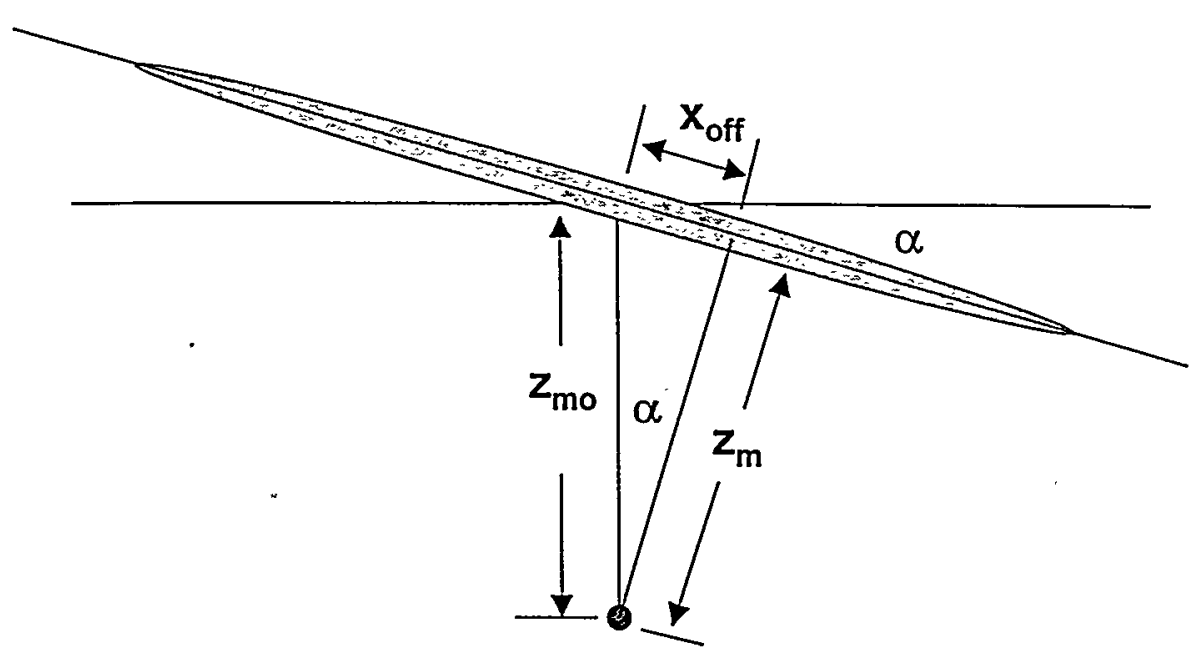

Figure 24. Schematic of monitoring distance and offset as a function of azimuth.

Figure 25 shows the effect of azimuth on the normal tilt distribution for a $30.5 \mathrm{~m}$ height and $305 \mathrm{~m}$ length fracture at $z_{m o}=152 \mathrm{~m}$ for several different azimuths in degrees. These results show that the tilt distribution hardly changes for azimuths less than $30^{\circ}$. This is both good and bad for tilt monitoring. It is good because the effect of an unknown azimuth is small for $\pm 30^{\circ}$ angles about the normal position, but it also makes it next to impossible to distinguish the azimuth with a single downhole array. In addition, the effect of azimuth for any angle is also indistinguishable from other combinations of length, height and pressure. Thus, the fracture azimuth must be known in order to accurately determine other fracture attributes with a single downhole tiltmeter array. 


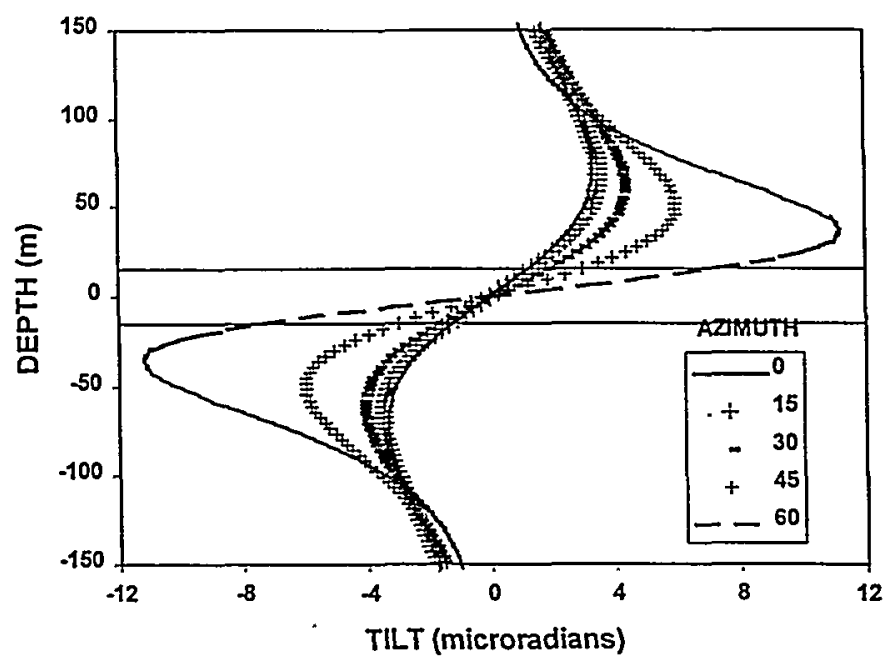

Figure 25. Effect of azimuth on normal tilt distribution for a $30.5 \mathrm{~m}$ height, $305 \mathrm{~m}$ length fracture at a monitor well separation distance of $152 \mathrm{~m}$.

Figure 26 shows the parallel tilt distributions for the same azimuth cases. As in most of the other results, the parallel tilt magnitudes are relatively small (unless the monitoring well is very close to the fracture) and contribute little to the total tilt magnitude. The parallel tilts are also not very helpful in determining the azimuth of the fracture.

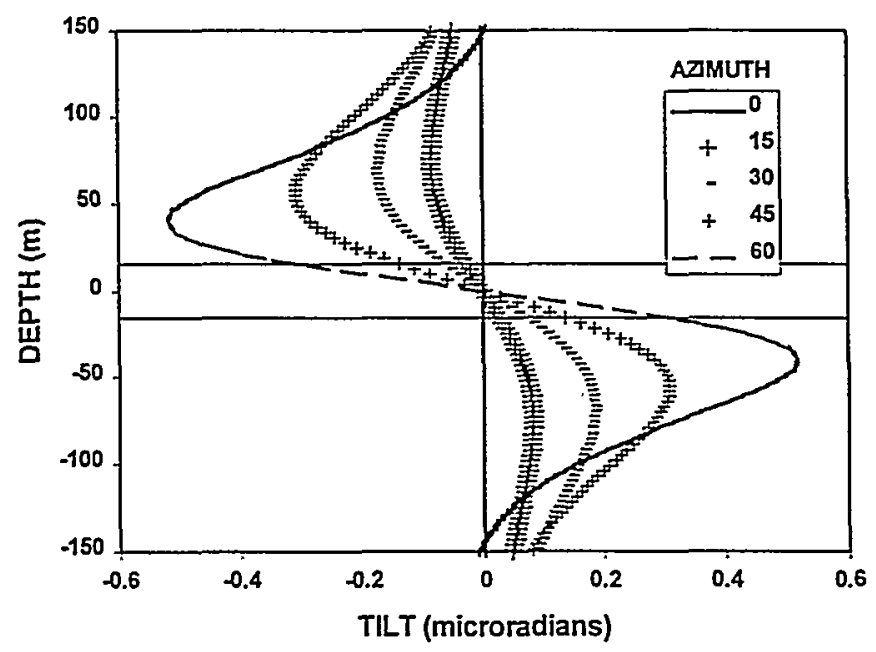

Figure 26. Effect of azimuth on parallel tilt distribution for a $30.5 \mathrm{~m}$ height, $305 \mathrm{~m}$ length fracture at a monitor-well separation distance of $152 \mathrm{~m}$. 
The effect of the crack shape, as a result of a variable stress distribution over the crack height, can be investigated using the layered-stress model based on England and Green's [1963] solution. The calculations were performed using the geometry of Figure 6 with $\mathrm{d}_{0}=30.5 \mathrm{~m}$ and $\mathrm{d}_{1}=\mathrm{d}_{2}=15.2 \mathrm{~m}$. For a uniform stress distribution across the fracture, $\sigma_{\text {top }}=\sigma_{\text {bottom }}=0$. For a symmetric case, $\sigma_{\text {top }}=\sigma_{\text {bottom }} \neq 0$.

The cases considered here are a symmetric case where a $61 \mathrm{~m}$ height fracture is given various stress contrasts while the tilts are calculated at a monitoring distance of $152 \mathrm{~m}$, and the nonsymmetric case where the bottom stress is kept at 3.45 MPa while the top stress is varied from 3.45 to $10.3 \mathrm{MPa}$. The symmetric results are shown in Figure 27 for stress contrasts of $0,3.45$, 6.89 and 10.3 MPa. The high stress layers have the effect of reducing the crack width and therefore reducing the induced deformation and tilt. The case for $10.3 \mathrm{MPa}$ is the maximum stress contrast that can be applied to a $61 \mathrm{~m}$ height fracture and still have it open over its entire length. For the other cases, the fracture height was artificially restricted to the $61 \mathrm{~m}$ height. The interesting feature of the high stress contrasts is they do not significantly change the location of the peaks (thus making the height estimate relatively reliable), but the large change in amplitude would give erroneous fracture lengths for a given pressure. 


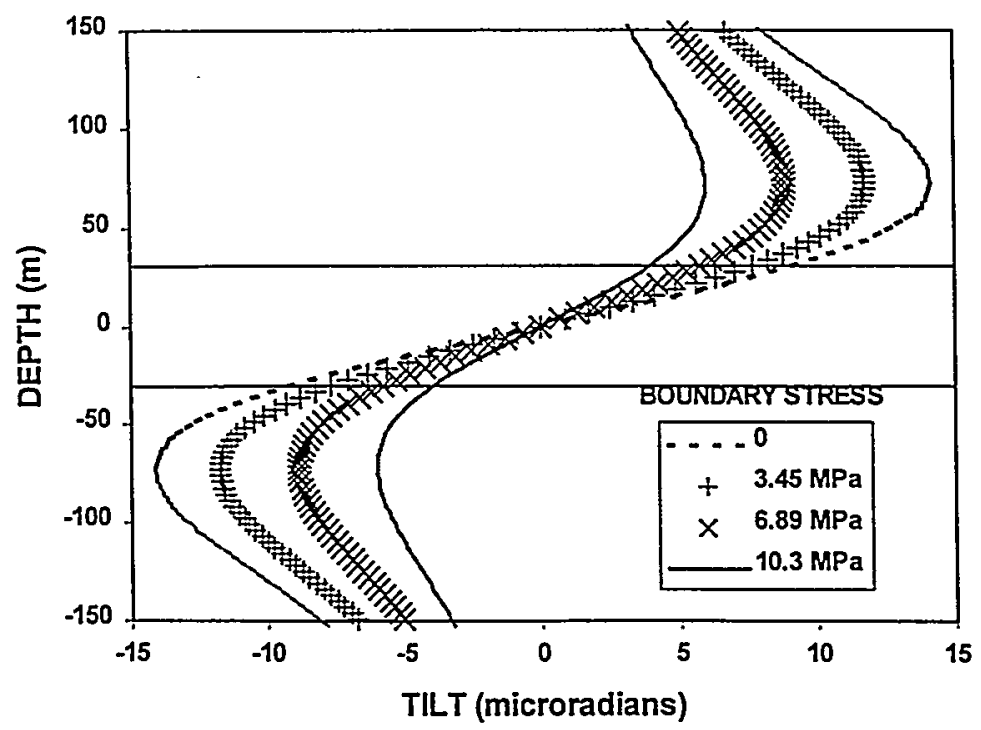

Figure 27. Effect of high stress layers on the normal tilt distribution due to reduced fracture width for a $61 \mathrm{~m}$ height fracture monitored $152 \mathrm{~m}$ away, 2D layered-stress case.

The non-symmetric case is shown in Figure 28 and is similar in many ways to the symmetric results in Figure 27. The cases shown are for a uniform stress (no contrasts), a symmetric contrast of 3.45 MPa, and non-symmetric contrasts of (a) 3.45 MPa below and 6.89 MPa above and (b) 3.45 MPa below and 10.3 MPa above. Even though the fracture shape is highly asymmetric, the tilt distribution is almost symmetric about the peaks and only shows a significant difference in the decay at the top and bottom. This is probably because the tilt peaks are most affected by the total width of the fracture, which affects both peaks in similar ways. Note that the symmetric case with $6.89 \mathrm{MPa}$ above and below is nearly the same as the nonsymmetric case with 3.45 $\mathrm{MPa}$ below and 10.3 MPa above (same net force on the fracture, just in different locations). 


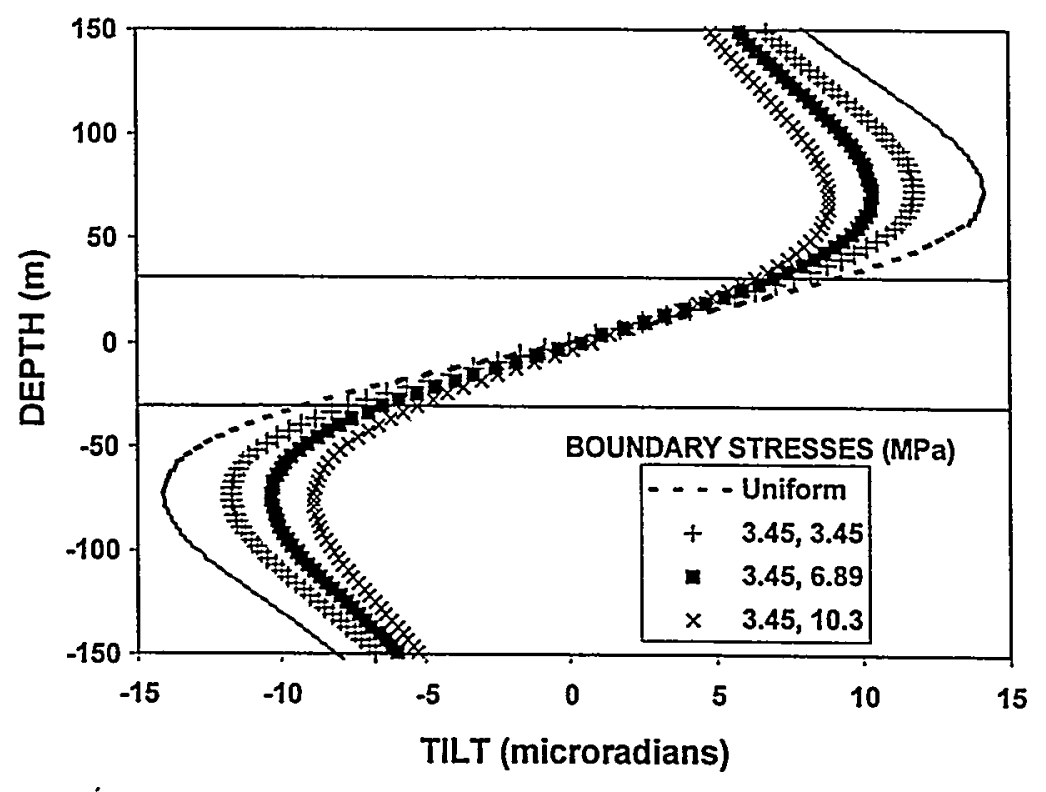

Figure 28. Effect of high stress layers on the normal tilt distribution due to reduced fracture width for a $61 \mathrm{~m}$ height fracture monitored $152 \mathrm{~m}$ away, 2D layered-stress case with asymmetric stresses.

\subsection{Comparison with Dislocation Solution}

The solution for a tensile dislocation of any size and orientation in a semi-infinite medium is given by Okada [1992]. This expression is very useful, but it is relatively lengthy and complicated. A simple expression for use with downhole tiltmeters can be developed by considering an infinitely long dislocation in an infinite medium. This solution will be accurate whenever the depth to the fracture is considerably larger than the height of the fracture and the depth to the shallowest monitoring station is relatively large. The expression for a tensile dislocation in an infinite medium can be extracted fairly easily using Maruyama's [1964] expressions as follows. 
The displacement due to any appropriate dislocation in an infinite medium is given by

$$
u_{m}(Q)=\iint_{\Sigma} \Delta u_{k}(P) T_{k l}^{m}(P, Q) n_{l}(P) d \Sigma
$$

where $u_{m}(Q)$ is the displacement of the $\mathrm{i}^{\text {th }}$ direction at the monitoring point $Q, \Delta u_{k}(P)$ is the opening (or other movement) of the dislocation at some point $P$ on the boundary $\Sigma, T_{k l}^{m}(P, Q)$ is the $k l$ component of stress at $P$ due to a unit body force in the $m$ direction located at $Q$, and $n_{l}(P)$ is the outward normal of the dislocation surface at the point P. Maruyama [1964] gives

$$
T_{k l}^{m}(P, Q)=\frac{1}{4 \pi}\left\{(1-\alpha)\left(-\delta_{k l} \frac{r_{m}}{r^{3}}+\delta_{m k} \frac{r_{l}}{r^{3}}+\delta_{l m} \frac{r_{k}}{r^{3}}\right)+3 \alpha \frac{r_{k} r_{l} r_{m}}{r^{5}}\right\},
$$

where $\delta$ is the Kronecker delta,

$$
\begin{gathered}
\alpha=\frac{1}{2(1-v)}, \\
r_{i}=x_{i}-\xi_{i},
\end{gathered}
$$

and

$$
r=\sqrt{\left(x_{1}-\xi_{1}\right)^{2}+\left(x_{2}-\xi_{2}\right)^{2}+\left(x_{3}-\xi_{3}\right)^{2}}
$$

for

$$
P=P\left(\xi_{1}, \xi_{2}, \xi_{3}\right), \quad Q=Q\left(x_{1}, x_{2}, x_{3}\right)
$$

For the simple case of a vertical dislocation of constant width, $b$, in the $x_{2}$ direction, then

$$
\Delta u_{k}=(0, b, 0) \text { and } n_{l}=(0,1,0)
$$

For downhole tiltmeters monitoring a $2 \mathrm{D}$ vertical fracture, only the vertical derivative of the normal displacement is needed and $T$ is simplified as 


$$
T_{22}^{2}=\frac{1}{4 \pi}\left\{(1-\alpha) \frac{r_{2}}{r^{3}}+3 \alpha \frac{r_{2}^{3}}{r^{5}}\right\}
$$

Inserting all of these parameters into the displacement integral, the displacement normal to the fracture is given by

$$
u_{2}(Q)=\frac{b}{4 \pi} \int_{H / 2}^{H / 2} d \xi_{1} \int_{L}^{L}\left[(1-\alpha) \frac{r_{2}}{r^{3}}+3 \alpha \frac{r_{2}^{3}}{r^{5}}\right] d \xi_{3}
$$

Performing the integrations and simplifying in the limit as $L \rightarrow \infty$, the normal displacement at some distance, $x_{2}$, for a $2 \mathrm{D}$ vertical fracture is given by

$$
\begin{aligned}
u_{2}(Q) & =\frac{b}{2 \pi}\left\{\tan ^{-1}\left[\frac{H / 2-x_{1}}{x_{2}}\right]+\tan ^{-1}\left[\frac{H / 2+x_{1}}{x_{2}}\right]\right\} \\
& +\frac{b \alpha}{2 \pi}\left\{\frac{\left(H / 2-x_{1}\right) x_{2}}{\left(H / 2-x_{1}\right)^{2}+x_{2}^{2}}+\frac{\left(H / 2+x_{1}\right) x_{2}}{\left(H / 2+x_{1}\right)^{2}+x_{2}^{2}}\right\}
\end{aligned}
$$

with $H$ being the fracture height, $x_{2}$ being the normal distance (horizontally) to the monitoring station, and $x_{1}$ being the vertical location of the monitoring station relative to the center of the fracture. Taking the derivative, the tilt at some position $\left(x_{1}, x_{2}\right)$ is

$$
\begin{aligned}
\frac{\partial u_{2}}{\partial x_{1}}(Q)= & \frac{b}{2 \pi}\left\{\frac{-x_{2}}{x_{2}^{2}+\left(H / 2-x_{1}\right)^{2}}+\frac{x_{2}}{x_{2}^{2}+\left(H / 2+x_{1}\right)^{2}}\right\} \\
& +\frac{b \alpha}{2 \pi}\left\{\frac{2 x_{2}\left(H / 2-x_{1}\right)^{2}}{\left[x_{2}^{2}+\left(H / 2-x_{1}\right)^{2}\right]^{2}}-\frac{2 x_{2}\left(H / 2+x_{1}\right)^{2}}{\left[x_{2}^{2}+\left(H / 2+x_{1}\right)^{2}\right]^{2}}\right\}
\end{aligned}
$$

This equation is a relatively simple expression that can easily be used to check results with the cracks equations. 
In comparing dislocation and crack models, one needs to find some basis to associate the crack pressure with the dislocation width. The most obvious solution is to equate the average displacement for both cases or, equivalently, the integrated displacement for both cases, assuming equal heights. Doing so for a $2 \mathrm{D}$ fracture results in a crack pressure given by

$$
\Delta P=\frac{4 E b}{2 \pi\left(1-v^{2}\right) H}
$$

If, for example, the dislocation width is $2.13 \mathrm{~cm}$, the equivalent pressure for a $61 \mathrm{~m}$ height fracture having the same material properties as the previous example would be $6.4 \mathrm{MPa}$. These values are used in the subsequent comparisons.

Figure 29 shows a comparison of the tilts from the dislocation model and the 2D crack model for a $61 \mathrm{~m}$ height fracture at a monitoring distance of $152 \mathrm{~m}$. At large distances, the dislocation and crack model are indistinguishable, as has been recognized previously [Davis, 1983].

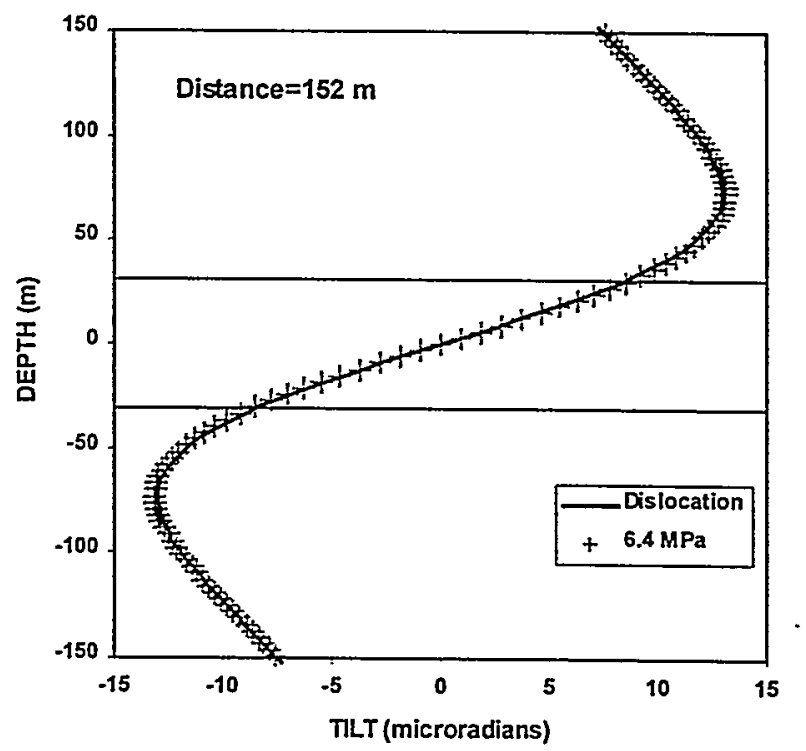

Figure 29. Comparison of crack and dislocation tilt magnitudes for a $61 \mathrm{~m}$ height fracture monitored at distance of $152 \mathrm{~m}$. 
If the monitoring distance is $61 \mathrm{~m}$, on the order of the crack height, the differences in the two models become apparent, as shown in Figure 30. The difference in the two models is about $6 \%$, but the shapes of the two curves are very similar and deductions about fracture height would be similar regardless of which model was used. For closer monitoring stations, as shown at $30.5 \mathrm{~m}$ in Figure 31, the discrepancy becomes greater and the location of the peak values for the two models begins to shift apart. The discrepancy in amplitude is about $10 \%$ and the shift in peak values is about $5 \mathrm{~m}$, which might lead one to deduce that the fracture height is $10 \mathrm{~m}$ less $(15 \%)$ than it actually is.

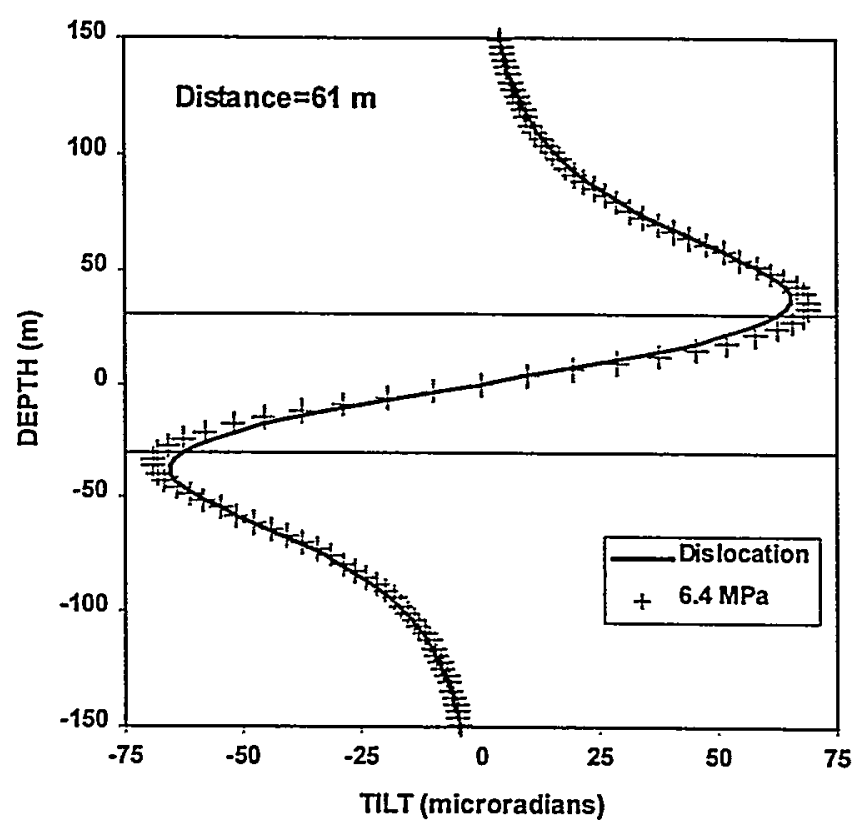

Figure 30. Comparison of crack and dislocation tilt magnitudes for a $61 \mathrm{~m}$ height fracture monitored at distance of $61 \mathrm{~m}$. 


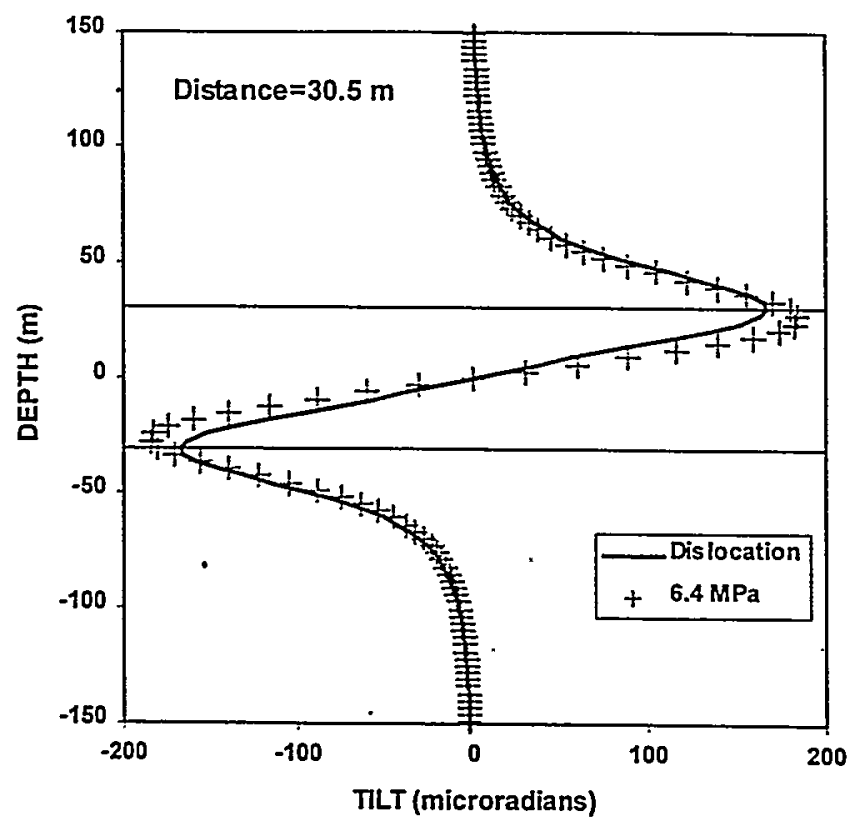

Figure 31. Comparison of crack and dislocation tilt magnitudes for a $61 \mathrm{~m}$ height fracture monitored at distance of $30.5 \mathrm{~m}$.

\subsection{Discussion and Conclusions}

The modeling results show that downhole tiltmeter arrays can provide data sufficiently accurate to estimate fracture height at distances up to 7-10 fracture heights for long fractures and 5 fracture radii for nearly circular fractures. This distance effect is due largely to the expanding tilt field with increasing distance from the fracture, the need for more extensive tiltmeter arrays, and the diminishing amplitude with distance.

The effect of dip on the tiltmeter results is somewhat complicated. A vertical fracture and a horizontal fracture produce the same shape of tilt field, but with different amplitudes. Fractures that are inclined only slightly from either the vertical or the horizontal have the effect of creating an asymmetry in amplitude and a shifting of the center of the fracture, but such effects could be masked by moduli changes in the various beds or various fracture irregularities. As a result, it is 
likely that small fracture dips could easily be interpreted as vertical offsets in the fracture locations. On the other hand, fractures inclined between $30^{\circ}$ and $60^{\circ}$ are quite easy to distinguish because of the single large amplitude peak.

In practice, the locations of the tilt peaks are often used as indicators of fracture height, but these results show that such an estimate can be seriously in error for situations where the monitoring distance is greater than the fracture height. In addition, this technique is not very accurate for penny-shaped fractures even if the fracture is close to the monitoring well.

Having a monitor array that is offset from the center of the fracture does not significantly erode the capabilities of the tilt measurement system. There is a loss in amplitude, but as long as. the monitoring position is not offset by more than 1.5 times the radius of the fracture (pennyshaped case), at least one-half the amplitude of the tilt remains.

The tilt component parallel to the fracture is generally a small percentage of the normal tilt unless the monitoring location is close to the fracture and offset from the center. The parallel component does provide additional information on fracture length and symmetry, but it can only be used if the tiltmeters are oriented [e.g.; Branagan et al., 1996].

The 3D-elliptic-crack calculations show that the $2 \mathrm{D}$ calculation is applicable when the length of the fracture is at least twice the monitoring distance. In general, it is difficult to extract accurate length information from a single tiltmeter array. The length and the offset position (equivalent of varying the fracture azimuth) both have similar effects and it is difficult to distinguish between the two under most situations. Also, the effect of increasing the fracture pressure is the same as increasing the length, at least on the normal tilt. To be able to extract accurate information, it would be helpful to use the parallel tilt component, but even this component would add limited information. One reasonable strategy for determining fracture 
length is to place the monitoring array close to the fracture but offset from the center by sizable percentage of the final fracture length. In such cases, it will be much easier to extract reliable information on fracture length on the wing by which the array is located. However, this strategy requires that the fracture azimuth and final length are known $a$ priori.

The effect of azimuth is relatively small for azimuths less than $30^{\circ}$ from the plane normal to the fracture-well/monitor-well line. This behavior simplifies analysis since small uncertainties in the azimuth will not cause large errors, but it also makes it impossible to extract accurate azimuth information from the tiltmeter data. As noted earlier, the effect of azimuth is very similar to that of length and pressure.

High stress layers above and below the fracture squeeze the width and reduce the tilt amplitudes, but they have only a small effect on the locations of the amplitude peaks. As a result, height can probably be accurately estimated in such circumstances, but the lower amplitude may lead to the conclusion that the length is shorter, the pressure is lower, or the fracture is closer (error in azimuth). Non-symmetric layers do not significantly change the amplitudes of the peaks and are difficult to distinguish relative to symmetric layers or other competing effects.

The dislocation calculation, which is widely used in tiltmeter analyses, is accurate if the distance to the monitoring well is at least 1.5 times the height for the 2D case. For closer monitoring stations, the dislocation analysis would result in an error in the fracture height and the difference in amplitude would suggest that the fracture is longer, wider, or closer.

In summary, these results show that a downhole tiltmeter array can be used to extract considerable useful information about hydraulic fractures and their growth processes. Nevertheless, there are so many variable parameters that any additional information provided by 
other diagnostics improves the accuracy of the tilt analysis. Perhaps the most effective way to improve the accuracy would be to use tiltmeter arrays in multiple wells and the development of oriented tiltmeters would serve to improve the analysis by allowing the independent use of both components of the tilt. Further work is needed to develop a better understanding of the effects of layering (both stress and modulus) on the resultant tilt field. Layering effects are likely to be the largest single source of error since the tilts are directly proportional to the modulus (pressurized crack solution) and the closure stress, and modulus variations of factors of 2-3 are common in sedimentary basins.

Acknowledgments. I would like to express my thanks for the support of the Gas Research Institute under contract 5089-211-2059, managed by Steve Wolhart, and the U.S. Department of Energy under contract DE-AC04-94AL85000 through the Natural Gas and Oil Technology Partnership.

\section{References}

Sandia is a multiprogram laboratory operated by Sandia Corporation, a Lockheed Martin Company, for the United States Department of Energy under contract DE-AC04-94AL85000.

Abramowitz, M. and I. A. Stegun, Handbook of Mathematical Functions, Dover Publications, New York, 1970.

Branagan, P. T., N. R. Warpinski, B. P. Engler, R. Wilmer, Measuring the hydraulic fractureinduced deformation of reservoir and adjacent rocks employing a deeply buried inclinometer array: GRI/DOE Multi-Site project, SPE 36451, Proceedings of the 1996 SPE Annual Tech. Conf. \& Exhib., Denver, CO, 327-335, Oct. 6-9, 1996.

Davis, P. M., Surface deformation associated with a dipping hydrofracture, Journal of Geophysical Research, 88, 5829-5834, July 10, 1983. 
England, A. H. and A. E. Green, Some two-dimensional punch and crack problems in classical elasticity, Proceedings of Cambridge Philosophical Society, 59, 489-500, 1963.

Green, A. E. and I. N. Sneddon, The distribution of stress in the neighbourhood of a flat elliptic crack in an elastic solid," Proc. Camb. Phil. Soc., 46, 159-163, 1950.

Green, A. E. and W. Zerna, Theoretical Elasticity, Dover Publications, New York, 274-278, 1968.

Maruyama, T., Statical elastic dislocations in an infinite and semi-infinite medium, Bulletin of the Earthquake Research Institute, Tokyo University, 42, 289-368, 1964.

Okada, Y., Internal deformation due to shear and tensile faults in a half-space, Bulletin of the Seismological Society of America, 82, No. 2, 1018-1040, April, 1992.

Peterson, R. E., S. L. Wolhart, K.-H. Frohne, N. R. Warpinski, P. T. Branagan and T. B. Wright, Fracture diagnostics research at the GRI/DOE Multi-Site project: overview of the concept and results, SPE 36449, Proceedings of the 1996 SPE Annual Tech. Conf. \& Exhib., Denver, CO, 315-325, October 6-9, 1996.

Pollard, D. D. and G. Holzhausen, On the mechanical interaction between a fluid-filled fracture and the earth's surface," Tectonophysics, 53, 27-57, 1979.

Sneddon, I. N. and H. A. Elliot, The opening of a Griffith crack under internal pressure, Quarterly of Applied Mathematics, 4, 262-267, 1946ä.

Sneddon, I. N., "The distribution of stress in the neighbourhood of a crack in an elastic solid, Proc. Royal Society A, 195, 229-260, $1946 \mathrm{~b}$.

Sih, G. C. and H. Liebowitz, Mathematical theories of brittle fracture, in Fracture, H. Liebowitz, ed., II, Academic Press, New York, p. 137, $196 \overline{8}$. 
Sun, R. J., Theoretical size of hydraulically induced horizontal fractures and corresponding surface uplift in an idealized medium, Journal of Geophysical Research, 74, 5995-6011, November 15, 1969.

Warpinski, N. R., HSTRESS: a computer program to calculate the height of a hydraulic fracture in a multi-layered stress medium, SAND89-1504, Sandia National Laboratories Report, Albuquerque, NM; July, 1989.

Warpinski,N. R., Interpretation of hydraulic fracture mapping experiments, SPE 27985, Proceedings of the Tulsa Centennial Petroleum Engineering Symposium, Tulsa, OK, 291-300, August 29-31, 1994.

Warpinski, N. R., Hydraulic fracture diagnostics, Journal of Petroleum Technology, 48, 907-910, October, 1996.

Warpinski, N. R., P. T. Branagan, R. E. Peterson, J. E. Fix, J. E. Uhl, B. P. Engler and R. Wilmer, Microseismic and deformation imaging of hydraulic fracture growth and geometry in the C sand interval, GRI/DOE M-Site project, SPE 38573, Proceedings of the 1997 SPE Annual Tech. Conf. \& Exhib., San Antonio, TX, 87-98, October 5-8, 1997 a.

Warpinski, N. R., P. T. Branagan, B. P. Engler, R. Wilmer and S. L. Wolhart, Evaluation of a downhole tiltmeter array for monitoring hydraulic fractures, Proceedings of the 36 th U.S. Rock Mechänics Symposium, Columbia University, NY, June 29 - July 2, $1997 \mathrm{~b}$. Westergaard, H. M., Bearing pressures and cracks, Journal of Applied Mechanics, 61, A-49 to A-53, June, 1939.

Whittaker, E. T. and G. N. Watson, A Course of Modern Analysis, Cambridge, 1927. Wright, C. A., Tiltmeter fracture mapping: from the surface and now downhole, Petroleum Engineer International, 50-56, January, 1998. 
Wright, C. A., E. J. Davis, G. M. Golich, J. F. Ward, S. L. Demetrius, W. A. Minner and L. Weijers, Downhole tiltmeter fracture mapping: finally measuring hydraulic fracture dimensions, SPE 46194, Proceedings of the SPE Western Regional Conf., Bakersfield, CA, May 10-13, 1998. 